CERN-EP/90-19

February $9^{\text {th }}, 1990$

\title{
Study of Hadronic Decays of the $Z^{0}$ Boson
}

DELPHI collaboration

\begin{abstract}
Hadronic decays of $\mathrm{Z}^{0}$ bosons are studied in the Delphi detector. Global event variables and single particle inclusive distributions are compared with QCD-based predictions. The mean charged multiplicity is found to be $20.6 \pm 1.0$ (stat+syst). The mean values of the sphericity, aplanarity, thrust, minor value, $p_{T}^{\text {in }}$ and $p_{T}^{\text {out }}$ are compared with values found at lower energy $\mathrm{e}^{+} \mathrm{e}^{-}$colliders.
\end{abstract}

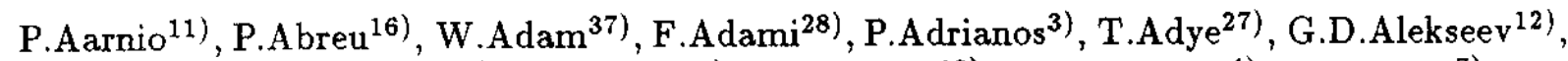
J.V.Allaby ${ }^{7)}$, P.Allen ${ }^{36)}$, P.Allport ${ }^{27)}$, S.Almehed ${ }^{19)}$, S.J.Alvsvaag ${ }^{4}$, U.Amaldi ${ }^{7)}$, E.Anassontzis ${ }^{3)}$, P.Antilogus ${ }^{15)}$, W.D.Apel ${ }^{13)}$, B.Asman ${ }^{32)}$, F.Astesan ${ }^{18)}$, C.Astor Ferreres ${ }^{30)}$, J.E.Augustin $^{15)}$, A.Augustinus ${ }^{7)}$, P.Baillon ${ }^{7)}$, F.Barao ${ }^{16)}$, G.Barbiellini ${ }^{34)}$, D.Yu.Bardin ${ }^{12)}$, S.Barlag ${ }^{15)}$, J.Barlow ${ }^{27)}$, A.Baroncelli ${ }^{29)}$, M.Barranco-Luque ${ }^{7}$, G.Barreira ${ }^{16)}$, O.Barring ${ }^{19)}$, W.Bart ${ }^{17)}$, M.J.Bates ${ }^{25)}$, M.Baubillier ${ }^{18)}$, K.H.Becks ${ }^{39)}$, C.J.Beeston ${ }^{25)}$, W.Bell ${ }^{7}$, I.Belokopytov $^{31)}$, P.Beltran ${ }^{9)}$, D.Benedic ${ }^{8}$, J.M.Benlloch ${ }^{36)}$, M.Berggren ${ }^{7)}$, D.Bertrand ${ }^{2)}$, S.Biagi $^{17)}$, F.Bianchi' ${ }^{33)}$, J.H.Bibby ${ }^{25)}$, M.Bilenky ${ }^{12)}$, P.Billoir ${ }^{6)}$, N.Bingefors ${ }^{35)}$, J.Bjarne ${ }^{19)}$, D.Bloch ${ }^{8)}$, P.N.Bogolubov ${ }^{12)}$, D.Bollini ${ }^{5)}$, T.Bolognese ${ }^{28)}$, M.Bonapart ${ }^{22)}$, P.S.L.Booth ${ }^{17)}$, M.Boratav ${ }^{18)}$, P.Borgeaud ${ }^{28)}$, G.Borisov ${ }^{31)}$, H.Borner ${ }^{25)}$, C.Bosio ${ }^{29)}$, O.Botner ${ }^{35)}$, B.Bouquet $^{15)}$, M.Bozzo ${ }^{10)}$, S.Braibant ${ }^{7)}$, P.Branchini ${ }^{29)}$, K.D.Brand ${ }^{39)}$, C.Bricman ${ }^{2)}$, R.C.A.Brown ${ }^{7)}$, N.Brummer ${ }^{22)}$, J.M.Brunet ${ }^{6)}$, L.Bugge ${ }^{24)}$, T.Buran ${ }^{24)}$, H.Burmeister ${ }^{7)}$, C.Buttar ${ }^{25)}$, J.A.M.A.Buytaert ${ }^{2)}$, G.Cabras ${ }^{34)}$, M.Caccia ${ }^{20)}$, M.Calvi ${ }^{20)}$,

A.J.Camacho Rozas ${ }^{30)}$, J.E.Campagne ${ }^{18)}$, A.Campion ${ }^{17)}$, T.Camporesi ${ }^{7)}$, V.Canale ${ }^{29)}$, L.Carroll $^{17)}$, C.Caso ${ }^{10)}$, E.Castelli ${ }^{34)}$, V.Castillo Gimenez ${ }^{36)}$, A.Cattai ${ }^{7}$, F.R.Cavallo ${ }^{5)}$, L.Cerrito $^{29)}$, G.Chadwick ${ }^{7)}$, P.Charpentier ${ }^{7}$, P.Checchia ${ }^{26)}$, G.A.Chelkov ${ }^{12)}$, L.Chevalier ${ }^{28)}$, C.Chiccoli5), P.V.Chliapnikov $^{31)}$, V.Chorowicz ${ }^{18)}$, R.Cirio ${ }^{33)}$, M.P.Clara ${ }^{33)}$, J.L.Contreras ${ }^{36)}$, R.Contri ${ }^{10)}$, F.Couchot ${ }^{15)}$, H.B.Crawley ${ }^{1)}$, D.Crennell ${ }^{27)}$, M.Cresti ${ }^{26)}$, G.Crosetti ${ }^{10)}$, N.Crosland ${ }^{25)}$, M.Crozon ${ }^{6}$, J.Cuevas Maestro ${ }^{30)}$, L.S.Curwen ${ }^{17)}$, E.Dahl-Jensen ${ }^{21)}$, B.D'Almagne ${ }^{15)}$, M.Dam ${ }^{7)}$, G.Damgaard ${ }^{21)}$, G.Darbo ${ }^{10)}$, E.Daubie ${ }^{2)}$, M.Davenport ${ }^{7)}$, A.De Angelis ${ }^{34)}$, M.De Beer ${ }^{28)}$, C.De Clercq ${ }^{2)}$, N.De Groot ${ }^{22)}$, C.De La Vaissiere ${ }^{18)}$, D.Delikaris ${ }^{7)}$, P.Delpierre ${ }^{6}$, L.Di Ciaccio ${ }^{29)}$, A.N.Diddens ${ }^{22)}$, H.Dijkstra ${ }^{7)}$, N.Dimitriou ${ }^{9)}$,

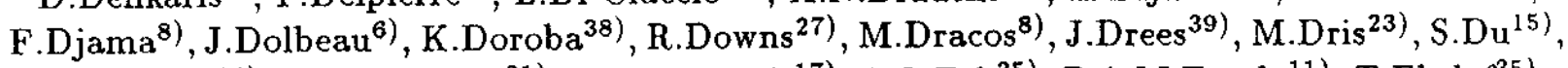
W.Dulinski ${ }^{14)}$, R.Dzhelyadin ${ }^{31)}$, D.N.Edwards ${ }^{17)}$, L.O.Eek ${ }^{35)}$, P.A.M.Eerola ${ }^{11)}$, T.Ekelof ${ }^{35)}$, G.Ekspong $^{32)}$, M.Ellila ${ }^{11)}$, J.P.Engel ${ }^{8)}$, V.Falaleev ${ }^{31)}$, A.Fenyuk ${ }^{31)}$, M.Fernandez Alonso ${ }^{30)}$, A.Ferrer $^{36)}$, S.Ferroni ${ }^{10)}$, T.A.Filippas ${ }^{23)}$, A.Firestone ${ }^{1)}$, H.G.Fischer ${ }^{7)}$, M.Flinn ${ }^{25)}$, H.Foeth ${ }^{7}$, E.Fokitis $^{23)}$, P.Folegati ${ }^{20)}$, F.Fontanelli ${ }^{10)}$, H.Forsbach ${ }^{39)}$, D.Fraissard ${ }^{7)}$, B.Franek ${ }^{27)}$,

K.E.Fransson ${ }^{35)}$, P.Frenkiel ${ }^{6)}$, D.C.Fries ${ }^{13)}$, A.G.Frodesen ${ }^{4)}$, R.Fruhwirth ${ }^{37)}$,

F.Fulda-Quenzer ${ }^{15)}$, J.Fuster ${ }^{7)}$, J.M.Gago ${ }^{16)}$, M.Gaillard ${ }^{15)}$, G.Galeazzi ${ }^{26)}$, D.Gamba $^{33)}$, 
C.Gaspar ${ }^{16)}$, U.Gasparini ${ }^{26)}$, P.Gavillet ${ }^{7)}$, S.Gawne ${ }^{17)}$, E.N.Gazis ${ }^{23)}$, J.F.Genat ${ }^{18)}$, J.P.Gerber ${ }^{8)}$, K.W.Glitza ${ }^{39)}$, R.Gokieli ${ }^{18)}$, V.M.Golovatyuk ${ }^{12)}$, P.Gomes ${ }^{16)}$, J.J.Gomez Y Cadenas ${ }^{36)}$, A.Goobar ${ }^{32)}$, G.Gopal2 ${ }^{27)}$, M.Gorbics ${ }^{1)}$, B.Goret ${ }^{7)}$, M.Gorski ${ }^{38)}$, G.Goujon ${ }^{28)}$, V.Gracco(0) A.Grant ${ }^{7)}$, F.Grard ${ }^{2)}$, E.Graziani ${ }^{29)}$, J.P.Grillet ${ }^{7)}$, M.H.Gros ${ }^{15)}$, M.Gros ${ }^{28)}$, G.Grosdidier ${ }^{15)}$, B.Grossetete ${ }^{18)}$, B.Grung ${ }^{4)}$, L.Guglielmi ${ }^{6)}$, S.Gumenyuk ${ }^{31)}$, J.Guy ${ }^{27)}$, F.Hahn ${ }^{39)}$, M.Hahn ${ }^{13)}$, S.Haider ${ }^{7)}$, J.Haissinski ${ }^{15)}$, Z.Hajduk $^{14)}$, A.Hakansson ${ }^{19)}$, A.Hallgren ${ }^{35)}$, K.Hamacher ${ }^{39)}$, G.Hamel De Monchenault ${ }^{28)}$, F.Harris ${ }^{25)}$, B.Heck ${ }^{7)}$, I.Herbst ${ }^{38)}$, J.J.Hernandez ${ }^{36)}$, P.Herquet ${ }^{2)}$, H.Herr ${ }^{7)}$, E.Higon ${ }^{36)}$, H.J.Hilke ${ }^{7)}$, T.Hofmokl ${ }^{38)}$,

S.O.Holmgren ${ }^{32)}$, J.E.Hooper ${ }^{21)}$, R.Horisberger ${ }^{7)}$, M.Houlden ${ }^{17)}$, A.Hrisoho ${ }^{15)}$, J.Hrubec ${ }^{37)}$,

K.Huitu ${ }^{11)}$, P.O.Hulth ${ }^{32)}$, K.Hultqvist ${ }^{32)}$, D.Husson ${ }^{8}$, B.D.Hyams ${ }^{7}$, D.Imbault ${ }^{18)}$, M.Innocente ${ }^{34)}$, P.Ioannou ${ }^{3)}$, P.S.Iversen ${ }^{4)}$, J.N.Jackson ${ }^{17)}$, P.Jalocha ${ }^{14)}$, G.Jarlskog ${ }^{19)}$, P.Jarry ${ }^{28)}$, B.Jean-Marie ${ }^{15)}$, J.Joensuu ${ }^{11)}$, E.K.Johansson ${ }^{32)}$, H.Johansson ${ }^{35)}$, S.Johansson ${ }^{19)}$, M.Jonker ${ }^{7)}$, P.Juillot ${ }^{8)}$, R.B.Kadyrov ${ }^{12)}$, G.Kalkanis ${ }^{3)}$, G.Kalmus ${ }^{27)}$, G.Kantardjian ${ }^{7)}$, S.Katsanevas ${ }^{3)}$, E.C.Katsoufis ${ }^{23)}$, R.Keranen ${ }^{11)}$, J.Kesteman ${ }^{2)}$, B.Khomenko ${ }^{12)}$, N.N.Khovanski ${ }^{12)}$, B.King ${ }^{17)}$, B.Kisielewski ${ }^{14)}$, H.Klein ${ }^{7)}$, W.Klempt ${ }^{7)}$, A.Klovning ${ }^{4)}$, B.Koene ${ }^{22)}$, P.Kokkinias ${ }^{9}$, I.Kontaxis ${ }^{3)}$, M.Kopf ${ }^{13)}$, M.Koratzinos ${ }^{7)}$, K.Korcyl ${ }^{14)}$, B.Korzen ${ }^{7)}$, P.Kostarakis ${ }^{9)}$, C.Kourkoumelis ${ }^{3)}$, T.Kreuzberger ${ }^{37)}$, J.Krolikowski ${ }^{38)}$, J.Krstic ${ }^{25)}$, U.Kruener-Marquis ${ }^{39)}$, W.Kucewicz ${ }^{20)}$, G.Kuhn ${ }^{7}$, K.Kurvinen ${ }^{11)}$, M.I.Laakso ${ }^{11)}$, C.Lambropoulos ${ }^{9)}$, L.Lanceri ${ }^{34)}$, D.Langerveld ${ }^{22)}$, V.Lapchine ${ }^{31)}$, V.Lapin ${ }^{31)}$, J.P.Laugier ${ }^{28)}$, R.Lauhakangas ${ }^{11)}$, P.Laurikainen ${ }^{11)}$, B.Lavigne ${ }^{15)}$, J.C.Le Grand ${ }^{7)}$, H.Lebbolo ${ }^{18)}$, G.Leder ${ }^{37)}$, J.Lemonne ${ }^{2)}$, G.Lenzen ${ }^{39)}$, V.Lepeltier ${ }^{15)}$, A.Letessier-Selvon ${ }^{18)}$, J.A.Lidbury ${ }^{27)}$, E.Lieb ${ }^{39)}$, E.Lillestol ${ }^{4)}$, E.Lillethun ${ }^{4)}$, I.Lippi ${ }^{26)}$, R.Llosa ${ }^{36)}$, B.Loerstad ${ }^{19)}$, M.Lokajicek ${ }^{12)}$, J.G.Loken ${ }^{25)}$, M.A.Lopez Aguera ${ }^{30)}$, P.Lorenz ${ }^{39)}$, D.Loukas ${ }^{8}$, R.Lucock ${ }^{27)}$, B.Lund-Jensen ${ }^{35)}$, P.Lutz ${ }^{6}$ ),

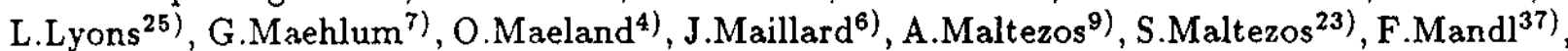
J.Marco $^{30)}$, J.C.Marin ${ }^{7}$, A.Markou ${ }^{9)}$, J.Mas ${ }^{6}$, L.Mathis ${ }^{6)}$, C.Matteuzzi ${ }^{20)}$, G.Matthiae ${ }^{29)}$, L.Mattsson ${ }^{35)}$, M.Mazzucato ${ }^{26)}$, M.Mc Cubbin'7), R.Mc Kay ${ }^{1)}$, E.Menichetti ${ }^{33)}$, C.Meroni ${ }^{20)}$, W.T.Meyer ${ }^{1)}$, M.Michael ${ }^{23)}$, J.Michalowski ${ }^{14)}$, W.A.Mitaroff ${ }^{37)}$, G.V.Mitselmakher ${ }^{12)}$, U.Mjoernmark ${ }^{19)}$, T.Moa ${ }^{32)}$, R.Moeller ${ }^{21)}$, K.Moenigig), M.R.Monge ${ }^{10)}$, P.Morettini ${ }^{10)}$,

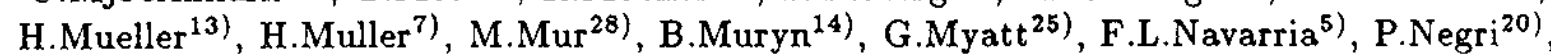
B.S.Nielsen ${ }^{21)}$, M.Nigro ${ }^{26)}$, V.Nikolaenko ${ }^{31)}$, M.Nonni ${ }^{29)}$, J.M.Noppe ${ }^{15)}$, M.Nordberg ${ }^{11)}$, S.Nounos ${ }^{3)}$, V.Obraztsov ${ }^{31)}$, T.Odegaard ${ }^{24)}$, R.Orava ${ }^{11)}$, A.Ouraou ${ }^{28)}$, J.Pagot ${ }^{15)}$, R.Pain ${ }^{18)}$, K.Pakonski ${ }^{14)}$, H.Palka ${ }^{14)}$, S.Palma Lopes ${ }^{18)}$, T.Papadopoulou ${ }^{23)}$, L.Pape ${ }^{7)}$, P.Pasini ${ }^{5)}$, M.Passeneau ${ }^{18)}$, A.Passeri ${ }^{29)}$, J.B.Pattison ${ }^{7}$, M.Pegoraro ${ }^{26)}$, V.Perevozchikov ${ }^{31)}$, J.Perez ${ }^{7)}$, M.Pernicka ${ }^{37)}$, A.Perrotta ${ }^{5}$, G.Petrucci ${ }^{7}$, T.Pettersen ${ }^{4}$, M.Pimenta ${ }^{18)}$, O.Pingot ${ }^{2)}$, C.Pinori ${ }^{26)}$, A.Pinsent ${ }^{25)}$, C.Poiret ${ }^{2)}$, M.E.Pol ${ }^{16)}$, G.Polok ${ }^{14)}$, P.Poropat ${ }^{34)}$, P.Privitera ${ }^{5}$, A.Pullia ${ }^{20)}$, J.Pyyhtia ${ }^{11)}$, P.Queru'), S.Quinton ${ }^{27)}$, A.A.Rademakers ${ }^{22)}$, D.Radojicic ${ }^{25)}$, S.Ragazzi ${ }^{20)}$, R.Ragazzon ${ }^{34)}$, W.H.Range ${ }^{17)}$, J.C.Raoul ${ }^{28)}$, P.Ratoff ${ }^{25)}$, A.Read ${ }^{24)}$, N.G.Redaelliid ${ }^{20}$, M.Regler ${ }^{37)}$, D.Reid ${ }^{17)}$, M.V.Reis ${ }^{16)}$, P.Renton ${ }^{25)}$, L.K.Resvanis ${ }^{3)}$, F.Richard ${ }^{15)}$, G.Rinaudo ${ }^{33)}$, A.M.Romaya ${ }^{25)}$, A.Romero ${ }^{33)}$, I.Roncagliolo ${ }^{10)}$, P.Ronchese ${ }^{26)}$, R.Rongved ${ }^{4)}$, E.Rosenberg ${ }^{1)}$, F.Rossel ${ }^{18)}$, E.Rosso ${ }^{7)}$, P.Roudeau ${ }^{15)}$, T.Rovelli ${ }^{5)}$, V.Ruhlmann ${ }^{28)}$, A.Ruiz ${ }^{30)}$, K.Rybicki ${ }^{14)}$, H.Saarikko ${ }^{11)}$, D.Sacco ${ }^{29)}$, Y.Sacquin ${ }^{28)}$, A.Sadovsky ${ }^{12)}$, C.W.Salgado ${ }^{36)}$, J.Salt ${ }^{36)}$, A.Samarin ${ }^{31)}$, E.Sanchez ${ }^{36)}$, E.Sanchis ${ }^{36)}$, M.Sannino ${ }^{10)}$, M.Schaeffer ${ }^{8}$, H.Schneider ${ }^{13)}$, F.Scuri ${ }^{34)}$, A.Sebastia ${ }^{36)}$, Y.V.Sedykh ${ }^{12}$, A.M.Segar ${ }^{25)}$, R.Sekulin ${ }^{27)}$, M.Sessa ${ }^{34)}$, G.Sette ${ }^{10)}$, R.Seufert ${ }^{13)}$, R.C.Shellard ${ }^{7)}$, P.Siegrist ${ }^{28)}$, S.Simonetti ${ }^{10)}$, F.Simonetto ${ }^{26)}$, A.N.Sissakian ${ }^{22)}$, T.B.Skaalii" ${ }^{24}$, J.Skeens ${ }^{1)}$, G.Skjevling ${ }^{24)}$, G.Smadja ${ }^{28)}$, G.R.Smith ${ }^{27)}$, R.Sosnowski ${ }^{38)}$; K.Spang ${ }^{21)}$, T.Spassov ${ }^{12)}$, P.Spentzouris ${ }^{3)}$, E.Spiriti ${ }^{29)}$, S.Squarcia ${ }^{10)}$, H.Staeck ${ }^{39)}$, C.Stanescu ${ }^{29)}$, G.Starropoulos ${ }^{9)}$, F.Stichelbaut ${ }^{2)}$, 
A.Stocchi $^{20)}$, J.Strauss ${ }^{37)}$, R.Strub ${ }^{8)}$, E.Sundell ${ }^{11)}$, M.Szczekowski $^{38)}$, M.Szeptycka ${ }^{38)}$, P.Szymanski ${ }^{38)}$, S.Tavernier ${ }^{2)}$, E.Tcherniaev ${ }^{31)}$, G.Theodosiou ${ }^{9)}$, A.Tilquin ${ }^{6)}$,

J.Timmermans ${ }^{22)}$, V.G.Timofeev ${ }^{12)}$, L.G.Tkachev ${ }^{12)}$, D.Z.Toet ${ }^{22)}$, S.Topp-Jorgensen ${ }^{25)}$, A.K.Topphol ${ }^{4)}$, L.Tortora ${ }^{29)}$, D.Treille ${ }^{7)}$, U.Trevisan ${ }^{10)}$, G.Tristram ${ }^{6)}$, C.Troncon ${ }^{20)}$, T.K.Truong ${ }^{15)}$, E.N.Tsyganov ${ }^{12)}$, M.Turala ${ }^{14)}$, R.Turchetta ${ }^{8)}$, M.L.Turluer ${ }^{28)}$, T.Tuuva ${ }^{11)}$, I.Tyapkin ${ }^{12)}$, M.Tyndel ${ }^{27)}$, F.Udo ${ }^{22)}$, S.Ueberschaer ${ }^{39)}$, O.Ullaland ${ }^{7}$, V.A.Uvarov ${ }^{31)}$, G.Valenti ${ }^{5)}$, G.W.Van Apeldoorn ${ }^{22)}$, P.Van Dam ${ }^{22)}$, W.K.Van Doninck ${ }^{2)}$, B.Van Eijk ${ }^{7}$, N.Van Eijndhoven ${ }^{7)}$, C.Vander Velde ${ }^{2)}$, J.P.Vanuxem ${ }^{7)}$, J.Varela ${ }^{16)}$, P.Vaz ${ }^{16)}$, G.Vegni ${ }^{20)}$, M.E.Veitch ${ }^{25)}$, E.Vela ${ }^{36)}$, J.Velasco ${ }^{36)}$, L.Ventura ${ }^{26)}$, W.Venus ${ }^{27)}$, L.Vertogradov ${ }^{12)}$, D.Vilanova ${ }^{28)}$, L.Viseu Melo ${ }^{16)}$, E.V.Vlasov ${ }^{31)}$, A.S.Vodopianov ${ }^{12)}$, M.Vollmer ${ }^{39)}$, G.Voulgaris $^{3)}$, M.Voutilainen ${ }^{11)}$, V.Vrba ${ }^{12)}$, H.Wahlen ${ }^{39)}$, C.Walck ${ }^{32)}$, F.Waldner ${ }^{34)}$, M.Wayne ${ }^{1)}$, P.Weilhammer ${ }^{7)}$, J.Werner ${ }^{39)}$, A.M.Wetherell ${ }^{7)}$, J.H.Wickens ${ }^{2)}$, W.S.C.Williams ${ }^{25)}$, M.Winter ${ }^{8)}$, G.Wormser ${ }^{15)}$, K.Woschnagg ${ }^{35)}$, N.Yamdagni ${ }^{32)}$, J.M.Yelton ${ }^{25)}$, A.Zaitsev ${ }^{31)}$, A.Zalewska ${ }^{14)}$, P.Zalewski ${ }^{38)}$, E.Zevgolatakos ${ }^{9)}$, G.Zhang ${ }^{39)}$, N.I.Zimin ${ }^{12)}$, M.Zito ${ }^{10)}$, R.Zitoun ${ }^{18)}$, R.Zukanovich Funchal ${ }^{6)}$, G.Zumerle ${ }^{26)}$ 
(Submitted to Physics Letter B)

${ }^{1}$ Ames Laboratory and Department of Physics, Iowa State University, AMES IA 50011, U. S. A.

${ }^{2}$ Physics Department, Univ. Instelling Antwerpen, Universiteitsplein 1, B-2610 WILRIJK.

IIHE, ULB-VUB, Pleinlaan 2, B-1050 BRUXELLES.

Service de Phys. des Part. Elém., Faculté des Sciences, Université de l'Etat Mons, Av. Maistriau 19, B-7000 MONS.

${ }^{3}$ Physics Laboratory, University of Athens, Solonos Str. 104, GR-10680 ATHENS.

1) Department of Physics, University of Bergen, Allégaten 55, N-5007 BERGEN.

5) Dipartimento di Fisica, Università di Bologna and INFN, Via Irnerio 46, I-40126 BOLOGNA.

'Collège de France, Lab. de Physique Corpusculaire, 11 pl. M. Berthelot, F-75231 PARIS CEDEX 5.

"CERN, CH-1211 GENEVA 23.

'Division des Hautes Energies, CRN - Groupe DELPHI, B.P. 20 CRO, F-67037 STRASBOURG CEDEX.

"Greek Atomic Energy Commission, Nucl. Research Centre Demokritos, P.O. Box 60228, GR-15310 AGHIA PARASKEVI.

10) Dipartimento di Fisica, Università di Genova and INFN, Via Dodecaneso 33, I-16146 GENOVA.

11'Dept. of High Energy Physics, University of Helsinki, Siltavuorenpenger 20 C, SF-00170 HELSINKI 17.

12 J Joint Institute for Nuclear Research, Dubna, Head Post Office, P.O. Box 79, 101000 MOSCOW, U.R.S.S.

13) Institut für Experimentelle Kernphysik, Universität Karlsruhe, Postfach 6980, D-7500 KARLSRUHE 1.

${ }^{14)}$ High Energy Physics Laboratory, Institute of Nuclear Physics, Ul. Kawiory 26 a, PL-30055 KRAKOW 30.

15) Université de Paris-Sud, Lab. de l'Accélérateur Linéaire, Bat 200, F-91405 ORSAY.

${ }^{10 /}$ LIP, Av. Elias Garcia 14 - 1e, P-1000 LISBOA CODEX.

${ }^{17}$ Department of Physics, University of Liverpool, P.O. Box 147, GB - LIVERPOOL L69 3BX.

${ }^{181}$ LPNHE, Universités Paris VI et VII, Tour 33 (RdC), 4 place Jussieu, F-75230 PARIS CEDEX 05.

${ }^{19)}$ Department of Physics, University of Lund, Sölvegatan 14, S-22363 LUND.

20) Dipartimento di Fisica, Università di Milano and INFN, Via Celoria 16, I-20133 MILANO.

21) Niels Bohr Institute, Blegdamsvej 17, DK-2100 COPENHAGUE 0.

22) NIKHEF-H, Postbus 41882, NL-1009 DB AMSTERDAM.

${ }^{23}$ 'National Technical University, Physics Department, Zografou Campus, GR-15773 ATHENS.

24) Physics Department, University of Oslo, Blindern, N-1000 OSLO 3.

201 Nuclear Physics Laboratory, University of Oxford, Keble Road, GB - OXFORD OX1 3RH.

${ }^{26)}$ Dipartimento di Fisica, Università di Padova and INFN, Via Marzolo 8, 1-35131 PADOVA.

${ }^{27}$ Rutherford Appleton Laboratory, Chilton, GB - DIDCOT OX11 OQX.

28) CEN-Saclay, DPhPE, F-91191 GIF-SUR-YVETTE CEDEX.

29) Istituto Superiore di Sanità, Ist. Naz. di Fisica Nucl. (INFN), Viale Regina Elena 299, I-00161 ROMA. Dipartimento di Fisica, Università di Roma II and INFN, Tor Vergata, I-00173 ROMA.

${ }^{30}$ 'Facultad de Ciencias, Universidad de Santander, av. de los Castros, E - 39005 SANTANDER.

31'Inst. for High Energy Physics, P.O. Box 35, Protvino, SERPUKHOV (Moscow Region), U.R.S.S.

${ }^{32)}$ Institute of Physics, University of Stockholm, Vanadisvägen 9, S-113 46 STOCKHOLM.

331 Istituto di Fisica Sperimentale, Università di Torino and INFN, Via P. Giuria 1, 1-10125 TORINO.

34) Dipartimento di Fisica, Università di Trieste and INFN, Via A. Valerio 2, I-34127 TRIESTE.

Istituto di Fisica, Università di Udine, I-33100 UDINE.

${ }^{38}$ ) Department of Radiation Sciences, University of Uppsala, P.O. Box 535, S-751 21 UPPSALA.

${ }^{30}$ Inst. de Fisica Corpuscular IFIC, Centro Mixto Univ. de Valencia-CSIC, Avda. Dr. Moliner 50, E-46100 BURJASSOT (Valencia).

${ }^{37}$ Institut für Hochenergiephysik, Öesterreich Akad. d. Wissensch., Nikolsdorfergasse 18, A-1050 VIENNE.

38) Inst. Nuclear Studies and, University of Warsaw, Ul. Hoza 69, PL-00681 WARSZAWA.

${ }^{30}$ 'Fachbereich Physik, University of Wuppertal, Postfach 100 127, D-5600 WUPPERTAL 1. 


\section{Introduction}

This letter presents a study of the general properties of charged particles produced in hadronic decays of the $Z^{0}$ boson observed in the DELPHI detector[1] during the first months of operation of the new $e^{+} e^{-}$storage ring LEP at centre of mass energies of 91.0-91.5 GeV. We compare measured distributions of global event shape variables and of inclusive single particle variables and the energy dependence of some characteristic mean values with the expectations of four $\mathrm{QCD}$-based fragmentation models computed using parameters pre-tuned to lower energy data.

\section{The Detector}

The measurements of charged particles used in this analysis were made in the Time Projection Chamber (TPC) in which tracks are reconstructed from up to 16 space points at radii $r=36.5$ to $106.2 \mathrm{~cm}$. The superconducting solenoid had been commissioned during the pilot run at its nominal magnetic field of $1.2 \mathrm{~T}$, but the data presented here were taken while it was being operated at only $0.7 \mathrm{~T}$. In this reduced magnetic field, the TPC space point precision is about $300 \mu \mathrm{m}$ in $r \phi$ and $900 \mu \mathrm{m}$ in $z$ (the resolution varies with $r \phi$ and $z$ and with the angle at which the track crosses the readout pad row). The momentum resolution was found to be $\delta p / p^{2}= \pm 0.02$ $(\mathrm{GeV} / \mathrm{c})^{-1}$. Points on neighbouring tracks could be distinguished only if they were separated by at least $15 \mathrm{~mm}$ in $r \phi$ or $z$. No differences in track-finding efficiency were observed between the data and the Monte Carlo simulation.

The trigger for hadronic events that was available throughout the data-taking was based on two independent sets of scintillation counters covering polar angles of $40^{\circ}-140^{\circ}$. The High-density Projection Chamber (HPC), which is located inside the solenoid and measures electromagnetic energy with high granularity, contains a scintillator layer behind the first 5 radiation lengths that was sensitive to electromagnetic showers above about $2 \mathrm{GeV}$. The Time-of-Flight (TOF) system was sensitive to minimum ionising particles and consists of a single layer of 172 scintillation counters located outside the HPC and the solenoid. The HPC and TOF counters were each arranged in two groups of four quadrants symmetrically on opposite sides of the crossing point. Various sub-triggers were formed from back-to-back and majority coincidences between the HPC and TOF sectors. The final HPC+TOF trigger was the $O R$ of these sub-triggers.

For part of the data an additional independent trigger sensitive to charged particles in the polar angle range $40^{\circ}-140^{\circ}$ reaching the front of the HPC was formed by a coincidence between the inner tracking detector (ID) and the outer tracking detector (OD). The ID is a cylindrical drift chamber covering polar angles of approximately $20^{\circ}-160^{\circ}$ at radii of $12-28 \mathrm{~cm}$. It comprises a jet chamber section providing $24 r \phi$ coordinates surrounded by 5 trigger layers providing both $r \phi$ and $z$ coordinates. In the OD, which covers polar angles of $40^{\circ}-140^{\circ}$ at radii of $197-208 \mathrm{~cm}$, 5 layers of drift tubes provide precise $r \phi$ coordinates and three of them also provide crude but fast $z$ information. The ID +OD trigger allowed the efficiency of the HPC and TOF sub-triggers to be measured from the data by recording the trigger pattern event by event. For hadronic events with a sphericity axis between $40^{\circ}$ and $140^{\circ}$, i.e. for the events used in this analysis (see below), the efficiency of the final HPC+TOF trigger alone was found to be over $98 \%$. The 
overall trigger efficiency for the data used here was further augmented by the ID + OD trigger and by other triggers that were available for parts of the data taking.

\section{Selection Criteria}

Only charged tracks were used in this analysis. The tracks were retained only if

(a) they extrapolated back to within $5 \mathrm{~cm}$ of the beam axis in $r$ and to within $10 \mathrm{~cm}$ of the nominal crossing point in $z$,

(b) their momentum $p$ was larger than $0.1 \mathrm{GeV} / \mathrm{c}$,

(c) their measured track length was above $50 \mathrm{~cm}$

(d) their polar angle was between $25^{\circ}$ and $155^{\circ}$.

Hadronic events were then selected by requiring that

(a) each of the two hemispheres $\cos \theta<0$ and $\cos \theta>0$ contained a total charged energy $E_{c h}=\Sigma E_{i}$ larger than $3 \mathrm{GeV}$, where $E_{i}$ are the particle energies (assuming the $\pi$ mass),

(b) the total charged energy seen in both hemispheres together exceeded $15 \mathrm{GeV}$,

(c) there were at least 5 charged tracks with momenta above $0.2 \mathrm{GeV} / \mathrm{c}$,

(d) the polar angle $\theta$ of the sphericity axis was in the range $40^{\circ}<\theta<140^{\circ}$.

The resulting data sample comprised 2073 events. The last cut ensured that the retained events were well contained inside the TPC. After all four cuts, events due to beam-gas scattering and to $\gamma \gamma$ interactions were reduced to below $0.1 \%$ of the sample. The largest background was due to $\tau^{+} \tau^{-}$events. From the Monte Carlo simulation this was calculated to be $0.24 \%$ of the sample.

\section{The QCD calculations and fragmentation models}

QCD-based Monte Carlo event generators for LEP data have been reviewed in detail recently[2]. They consist of two parts: a perturbative phase, in which quarks and gluons are generated according to perturbative QCD, and a non-perturbative phase, which describes the transition from partons to hadrons. During the non-perturbative 'confinement' phase, the QCD coupling constant becomes so large that perturbation theory breaks down and one must resort to phenomenological models, of which the string fragmentation model from the Lund group[3] has been particularly successful. In this model the hadrons are formed along colour flux tubes or 
strings stretched between the outgoing partons. The model introduces explicit correlations between the outgoing hadrons which are experimentally testable and have been found to be in excellent agreement with lower energy data[2].

As an alternative to the Lund Monte Carlo we have used the Marchesini-Webber Monte Carlo[4], in which partons are first combined into colourless clusters which then either decay isotropically, if they have small masses, or, if the masses are larger, are split according to a special cluster algorithm which is analogous to string fragmentation. In this Monte Carlo the perturbative phase is based on the production of quark pairs followed by multiple gluon emission and the splitting of gluons into quark or gluon pairs. For the evolution of such parton showers the Altarelli-Parisi equations are used, which are based on the leading log approximation. After a certain minimum invariant mass of the partons has been reached, the non-perturbative phase takes over. In the Marchesini-Webber Monte Carlo we have used the default values of all free model parameters.

In the Lund Monte Carlo the perturbative phase can be generated either according to parton showers, or according to the exact second order matrix element (ME). The latter allows for at most four partons in the final state. All the higher order graphs have to be mimicked by the parametrization of the non-perturbative phase. But the advantage is that the 3 -jet cross section is correct, including the loop corrections, in contrast to most of the parton shower models based on the leading log approximation. For example, in the Lund parton shower option the 3 -jet rate has been weighted by the first order QCD matrix element in order to give the correct 3-jet rate.

The Lund Monte Carlo version Jetset 6.3 uses the Gutbrod-Kramer- Schierholz (GKS) matrix element[5]. This is known to neglect some small terms. Therefore we have tried in addition the Ellis-Ross-Terano (ERT) matrix element[6] as implemented by N. Magnussen[7], which has been found to agree with the ERT matrix element as implemented in the newest Lund version Jetset 7.2. In this case we used an 'optimized' scale in the definition of the coupling constant, ie $\alpha$, was evaluated with $\Lambda_{\overline{M S}}=120 \mathrm{MeV}$ at $\mu^{2}=0.005 Q^{2}$. This small scale (of the order of a few $\mathrm{GeV}$ ) implies a correspondingly large value of $\alpha$, and was optimised to describe jet multiplicities at lower energies $[7,8,9,10]$ correctly. The scale of $\alpha$, for the parton shower option is roughly the transverse momentum of the branching, which is also typically of the order of a few $\mathrm{GeV}$. The string fragmentation model parameters we have used are those determined by A. Petersen et al.[10] by tuning the model to data at a centre of mass energy of $29 \mathrm{GeV}$.

\section{Definitions of the variables}

The properties of the events are analysed in terms of commonly used global event shape variables. One set of these is

- the sphericity $S=\frac{3}{2}\left(Q_{1}+Q_{2}\right)$, which lies in the range $0<S<1$ and approaches 0 for thin 2-jet events and 1 for spherical events;

- the aplanarity $A=\frac{3}{2} Q_{1}$, which lies in the range $0<A<0.5$ and approaches 0 for planar events; 
- the variables $Q_{3}-Q_{2}$ and $Q_{2}-Q_{1}$;

where $Q_{1}+Q_{2}+Q_{3}=1$ and $Q_{1}<Q_{2}<Q_{3}$ are the normalised ordered eigenvalues of the momentum tensor $M_{\alpha \beta}=\Sigma_{j} p_{\alpha j} p_{\beta j}$ where $\alpha$ and $\beta$ refer to the $x, y$ and $z$ directions and the sum extends over all the charged particles in the event. The eigenvector $\mathbf{n}_{\mathbf{3}}$ defines the sphericity axis and $\mathbf{n}_{3}$ and $\mathbf{n}_{2}$ define the event plane. Because the momentum tensor uses the momenta quadratically, these variables are particularly sensitive to the high momentum particles in the event.

Alternatives to the above variables that use momenta linearly, and are therefore more sensitive to the low momentum particles and less sensitive to decays, are:

- the thrust value $T=\Sigma\left|p_{\| i}\right| / \Sigma\left|p_{i}\right|$, where $p_{\|}$refers to the momentum component along the axis for which the value of $T$ is maximal, called the thrust axis;

- the major value $M=\Sigma\left|p_{\| i}\right| / \Sigma\left|p_{i}\right|$, a thrust-like parameter where $p_{\|}$refers to the momentum component along the axis perpendicular to the thrust axis that gives the largest value of $M$, called the major axis; and the similarly defined minor value $m$ where $p_{\|}$ refers to the momentum component along the so-called minor axis which is perpendicular both to the thrust axis and to the major axis;

- the oblateness $O=M-m$, the difference between the major and minor values.

We have also compared the predictions of the models with the distributions of single charged particles in the inclusive variables

- the rapidity $y_{\pi}=\frac{1}{2} \ln \left(\frac{\left(E+p_{\| \mid}\right)}{\left(E-p_{\| \mid}\right)}\right)$, where $p_{\| \mid}$refers to the momentum component parallel to the thrust axis and the $\pi$ mass is assumed.

- the momentum-fraction variable $x_{p}=2 p / \sqrt{S}=p / p_{\text {beam }}$,

- the momentum components transverse to the sphericity axis and in and out of the event plane, $p_{T}^{\text {in }}$ and $p_{T}^{\text {out }}$.

\section{Corrections and systematic errors}

Monte Carlo simulations were used to correct the event shape variable distributions and individual particle distributions for the geometrical acceptance and kinematical cuts, the detector resolution, acceptance inefficiencies, particle interactions in the material of the detector, other detector imperfections and the effects of radiated photons. The Lund parton-shower model (Jetset 6.3) was used to generate $25000 \mathrm{Z}^{0}$ events decaying to pairs of $u, d, c, s, b$ quarks. A correction factor $\mathrm{C}(\mathrm{x})$ for each bin in each real data plot was then obtained by comparing the bin occupancy at the beginning of the simulation (the 'true' distribution) with the bin occupancy after reconstruction and selection (the 'observed' distribution):

$$
C(x)=\left(\frac{1}{N} \frac{\mathrm{dn}}{\mathrm{dx}}\right)_{\text {true }} /\left(\frac{1}{\mathrm{~N}} \frac{\mathrm{dn}}{\mathrm{dx}}\right)_{\text {observed }}
$$


The 'true' distributions were constructed from the final state particles of lifetime above $10^{-9}$ seconds in events generated without initial state radiation that had not yet been tracked through the detector. The 'observed' distributions were constructed from the final state particles observed after tracking events generated with initial state radiation through the Delphi detector with reduced field to produce simulated raw data which were then processed through the same reconstruction and analysis programs as the real data.

The value of the correction factor $\mathrm{C}$ lies between 0.7 and 1.3 for all the data points of the global event shape variable distributions and the $p_{T}^{\text {in }}$ and $p_{T}^{\text {out }}$ distributions, and between 0.6 and 1.6 for all points of the $x_{p}$ and $y_{\pi}$ distributions.

The correction factor $\mathrm{C}$ was also evaluated using the Marchesini-Webber parton shower model and the Lund GKS matrix element model. The variance of the $C(x)$ values computed from the three different models was taken as one contribution to the systematic uncertainty. Other contributions arise from possible differences between the actual detector performance and that represented in the simulation program. To evaluate these, we tested the effects of a range of possible differences, such as additional momentum smearing, a constant sagitta shift and a different drift velocity inside the TPC, in the Monte Carlo simulation. We also varied our selection criteria over a wide range. These studies indicated that for all the data points presented below, the total systematic uncertainty is less than or roughly equal to the errors shown.

\section{Results}

The corrected measured global event variable distributions deduced from the charged tracks are compared with the Monte Carlo predictions in Figs. 1 and 2 and the inclusive single charged particle distributions are shown in Fig. 3. The horizontal bars indicate the corresponding bin width, the vertical bars show the statistical errors.

Both the Lund parton shower model and the Marchesini-Webber parton shower model describe well the shapes of all the measured distributions. However, the measured distributions of aplanarity, minor value and $p_{T}^{\text {out }}$, which are sensitive to multigluon emission, are significantly better reproduced by the Lund parton shower model. The data are less well described by the other two event generators, based on QCD matrix elements up to second order in $\alpha_{s}$, since they contain energy dependent model parameters tuned to PETRA/PEP data. The ERT matrix element generator with modified scale describes the tails of the aplanarity, minor value and $p_{T}^{\text {out }}$ distributions somewhat better than the GKS matrix element generator without modified scale.

The reason why the parton shower models can describe the data better than the matrix element models over a wide energy range without retuning of parameters is clear: the softening of the fragmentation function due to the larger phase space for multiple gluon emission is implemented, via the Altarelli-Parisi evolution equations, only in the parton shower models. In the matrix element generators it has to be put in by hand. After such retuning of the fragmentation parameters[11], the agreement with all the data distributions becomes excellent, as shown for the rapidity distribution in Fig. 3a.

The fully corrected mean values obtained for the quadratic momentum-tensor shape variables 
are $\langle S\rangle=0.073 \pm 0.004,\langle A\rangle=0.0121 \pm 0.0014$ and for the linear thrust-like variables $<T>=0.934 \pm 0.003,<m>=0.096 \pm 0.003$. The mean transverse momentum values are $\left\langle p_{T}^{\text {in }}>=0.434 \pm 0.014 \mathrm{GeV} / \mathrm{c},\left\langle p_{T}^{\text {out }}>=0.231 \pm 0.007 \mathrm{GeV} / \mathrm{c}\right.\right.$. The corrections to these mean values include a correction for missing neutral particles. For this purpose, we computed the 'true' distributions of charged and neutral particles, the numerator of the correction factors $\mathrm{C}(\mathrm{x})$, using the Lund parton shower generator. The size of the correction is less than $1 \%$ for $\langle S\rangle,\langle A\rangle$ and $\langle T\rangle, 3 \%$ for $\langle m\rangle, 1.3 \%$ for $\left\langle p_{T}^{\text {in }}\right\rangle$ and $4 \%$ for $\left\langle p_{T}^{\text {out }}\right\rangle$.

The measured mean multiplicity of charged particles was obtained by integrating the rapidity distribution. After a $2 \pm 1 \%$ correction for electrons from photon conversions before the TPC that were not accounted for in the Monte Carlo, we find $\left\langle n_{c h}\right\rangle=20.6 \pm 1.0$. The corresponding Monte Carlo predictions are 21.1 for the Lund parton shower model, 21.0 for the MarchesiniWebber parton shower model, 18.0 for the GKS model, 18.2 for the ERT matrix element model with modified scale, and 21.2 for the matrix element model with retuned parameters[11].

The quoted errors of all the mean values contain the statistical error of the data and the systematic errors added quadratically. The results agree well with other results obtained at this energy $[12,13]$.

The energy dependence of the mean values is shown in Fig. 4 where the data from this experiment are plotted together with the HRS[14], TASSO[15], AMY[16] and Mark II[10, 12] results as a function of the centre of mass energy. The curves represent the model predictions keeping, in each case, all free parameters constant. While the overall trends of the data are roughly reproduced by all models, there are important differences in detail. The Lund parton shower model gives the best description for $\langle S\rangle,\langle A\rangle,\langle T\rangle$ and $\langle m\rangle$. Both matrix element models predict a stronger energy dependence of $\langle A\rangle$ and $\langle m\rangle$ than is observed. The increase in $\left\langle p_{T}^{\text {in }}\right\rangle$ with energy is smaller in the data than in all the models.

\section{Summary and Conclusions}

We have measured global event shape variable distributions and single particle inclusive distributions of charged particles produced in hadronic decays of $\mathrm{Z}^{0}$ bosons in the Delphi detector during the first months of operation at LEP. The measured mean charged multiplicity is $20.6 \pm 1.0$. We have compared the data with a range of Monte Carlo predictions for $\mathrm{Z}^{\hat{0}} \rightarrow q \bar{q}$ decays of fragmentation models based on perturbative QCD. The best overall agreement is obtained with the Lund parton shower model with string fragmentation. The Marchesini-Webber model agrees less well with some distributions, particularly the distributions in aplanarity, minor value and $p_{T}^{\text {out }}$, which are sensitive to multigluon effects. The models using QCD matrix elements to second order in $\alpha_{s}$ adjusted to PETRA/PEP data need retuning of the model parameters at LEP energies.

We have also evaluated the mean values of a selection of these variables, including corrections for missing neutral particles, and compared them with data obtained from other experiments, mostly at lower energies. The trend of the energy dependence of the mean sphericity, aplanarity and minor value is well predicted by the Lund parton shower model. However, the rise of $\left\langle p_{T}^{\text {in }}\right\rangle$ with energy is observed to be smaller than predicted. 
Acknowledgements: We are greatly indebted to our technical staffs and collaborators and funding agencies for their support in building the Delphi detector and to the many members of LEP Division for the speedy commissioning and superb performance of the LEP machine. 


\section{List of Figures}

1 Observed distributions of the quadratic global event variables (a) sphericity, (b) aplanarity, (c) $Q_{3}-Q_{2}$ and (d) $Q_{2}-Q_{1}$ compared with Monte Carlo predictions. The full curve corresponds to the Lund parton shower model (Jetset 6.3), the broken curve to the Marchesini-Webber parton shower model (Herwig 3.4), and the dotted and dashed curves to Lund with the GKS and ERT second order matrix elements respectively (in all cases using parameter values optimised at PETRA/PEP energies $) \ldots \ldots \ldots \ldots \ldots \ldots$

2 Observed distributions of the linear global event variables (a) thrust, (b) major value, (c) minor value and (d) oblateness compared with Monte Carlo predictions (curves as in Fig. 1) . . . . . . . . . . . . . . . . . . . . . 1

3 Observed distributions of the single charged particle inclusive variables (a) rapidity $y_{w}$, (b) momentum fraction $x_{p}=p / p_{\text {beam }}$, (c) transverse momentum component in the event plane $p_{T}^{\text {in }}$ and (d) transverse momentum component out of the event plane $p_{T}^{\text {out }}$ compared with Monte Carlo predictions (the dot-dash curve in (a) represents the ERT model after a retuning of the fragmentation parameters, the other curves are as in Fig. 1) . . . . . . . . . . . . . .

4 Energy dependence of the average values of (a) sphericity $\langle S\rangle$, (b) aplanarity $\langle A\rangle$, (c) thrust $\langle T\rangle$, (d) minor value $\langle m\rangle$, (e) $p_{T}^{\text {in }}$ (f) $p_{T}^{\text {out }}$ corrected for missing neutrals compared with Monte Carlo predictions (curves as in Fig. 1). 


\section{References}

[1] a) Technical Proposal, DELPHI 83-66/1,

b) Phys. Lett. 231B (1989) 539,

c) 'The Delphi Detector', paper in preparation, to be submitted to Nuclear Instruments and Methods.

[2] QCD generators for LEP, Proc. of the 1989 Workshop on Z Physics at LEP 1, B. Bambah et al., CERN 89-08, Vol. 3, p.143, Eds. G. Altarelli, R. Kleiss and C. Verzegnassi.

[3] T. Sjöstrand, Comp. Phys. Comm. 27 (1982) 243, ibid. 28 (1983) 229.

T. Sjöstrand and M. Bengtsson, Comp. Phys. Commun. 43 (1987) 367.

[4] G. Marchesini and B.R. Webber, Nucl. Phys. B238 (1984) 1.

[5] F. Gutbrod, G. Kramer and G. Schierholz, Z. Phys. C21 (1984) 235.

[6] R.K. Ellis, D.A. Ross and E.A. Terrano, Nucl. Phys. B178 (1981) 421.

[7] N. Magnussen, thesis, Univ. of Wuppertal, 1988; DESY F22-89-01; (we used the p scheme of parton recombination).

[8] S. Bethke, LBL-26958, (1989).

[9] JADE Coll., W. Bartel et al., Z. Phys. C33 (1986) 23.

[10] Mark II Coll., A. Petersen et al., Phys. Rev. D37 (1988) 1.

[11] W. de Boer, H. Fürstenau and J. Köhne, to be published.

[12] Mark II Coil., G.S. Abrams et al., Phys. Rev. Lett. 63 (1989) 1558.

[13] ALEPH Coll., D. Decamp et al., Phys. Lett. 234B (1990) 209.

[14] HRS Coll., D. Bender et al., Phys. Rev. D31 (1985) 1.

[15] TASSO Coll., M. Althoff et al., Z. Phys. C22 (1984) 307; and W. Braunschweig et al., Z. Phys C41 (1988) 359.

[16] AMY Coll., Y. K. Li et al., KEK preprint 89-149. 


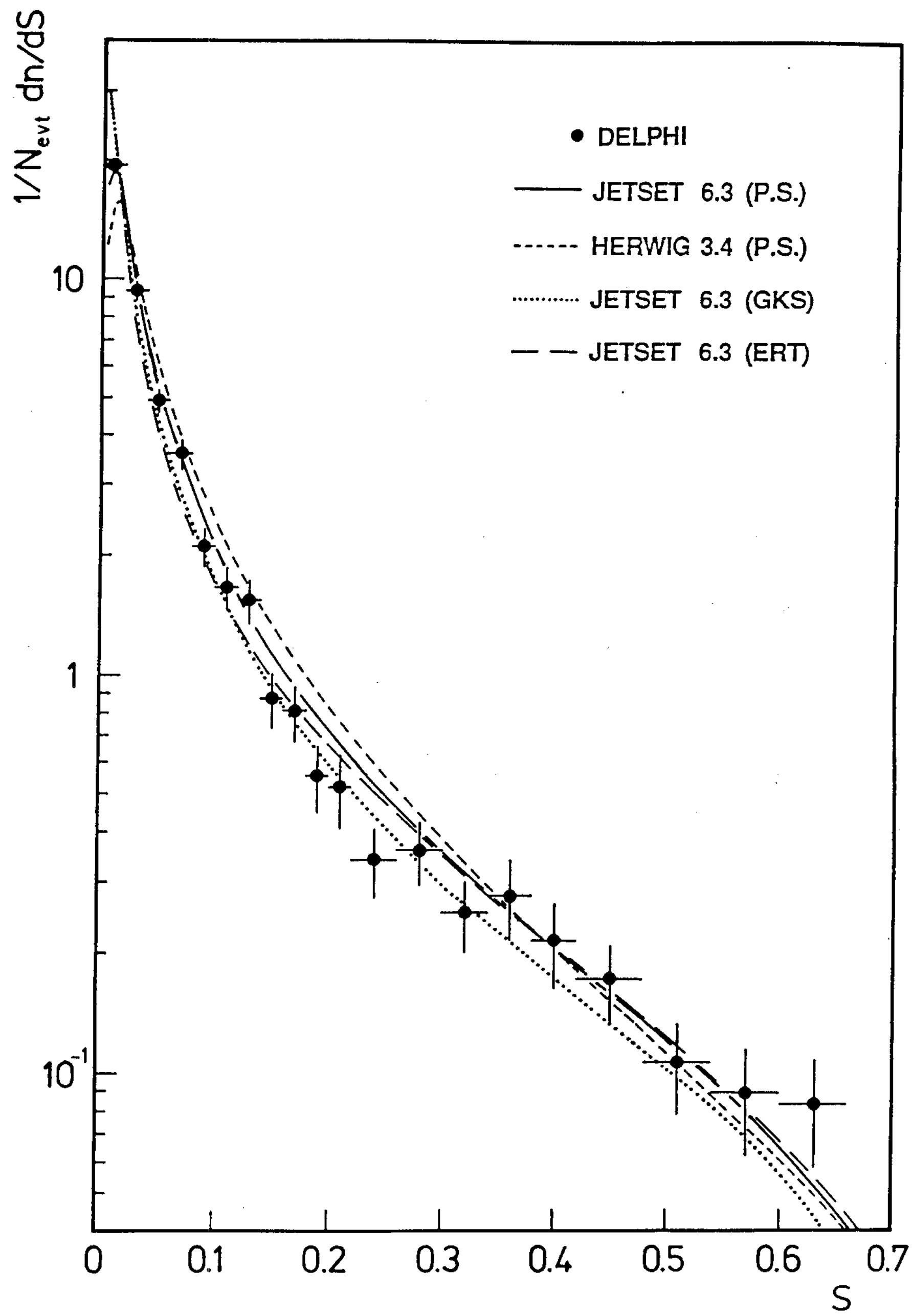

Fig. $1(a)$ 


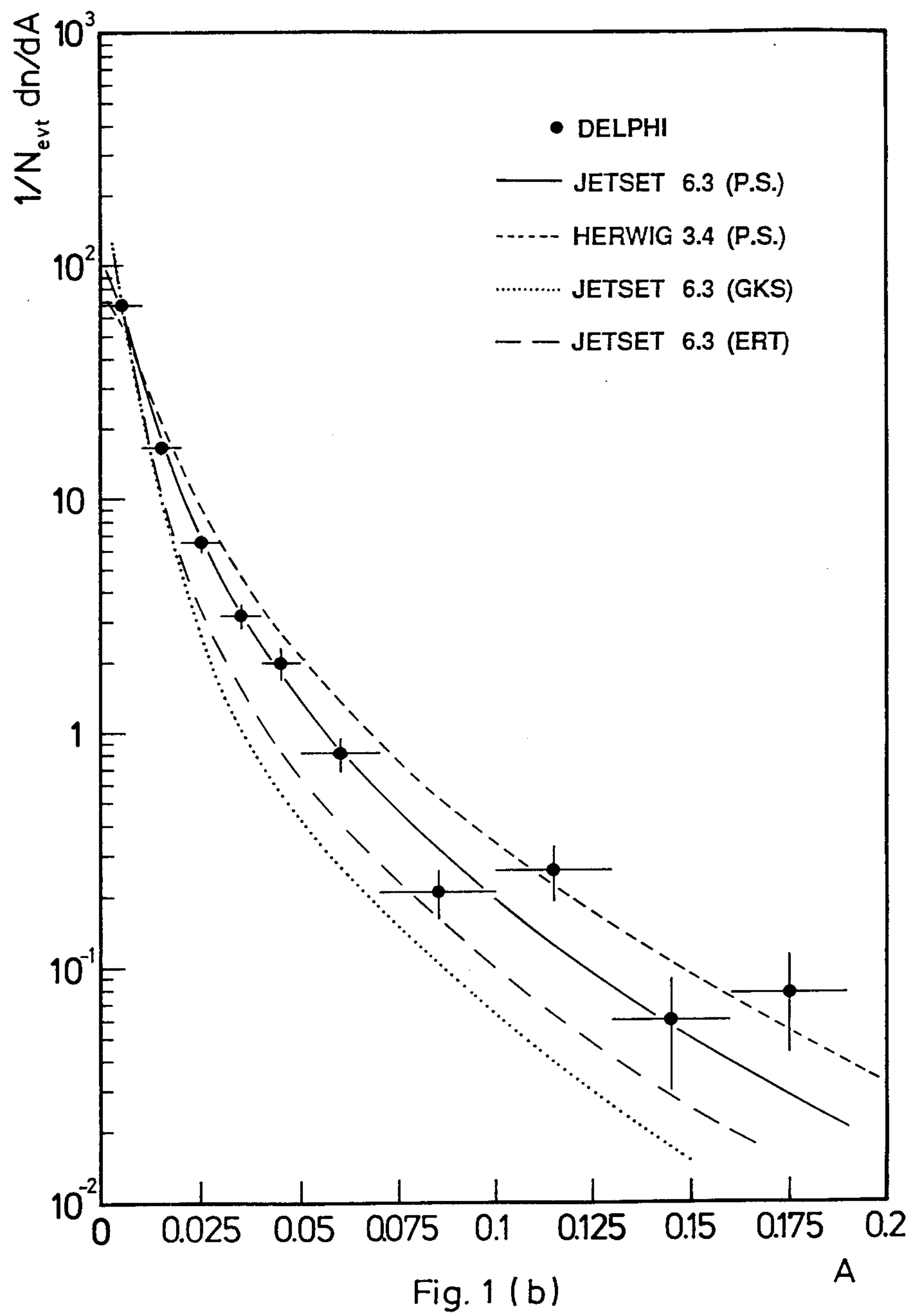




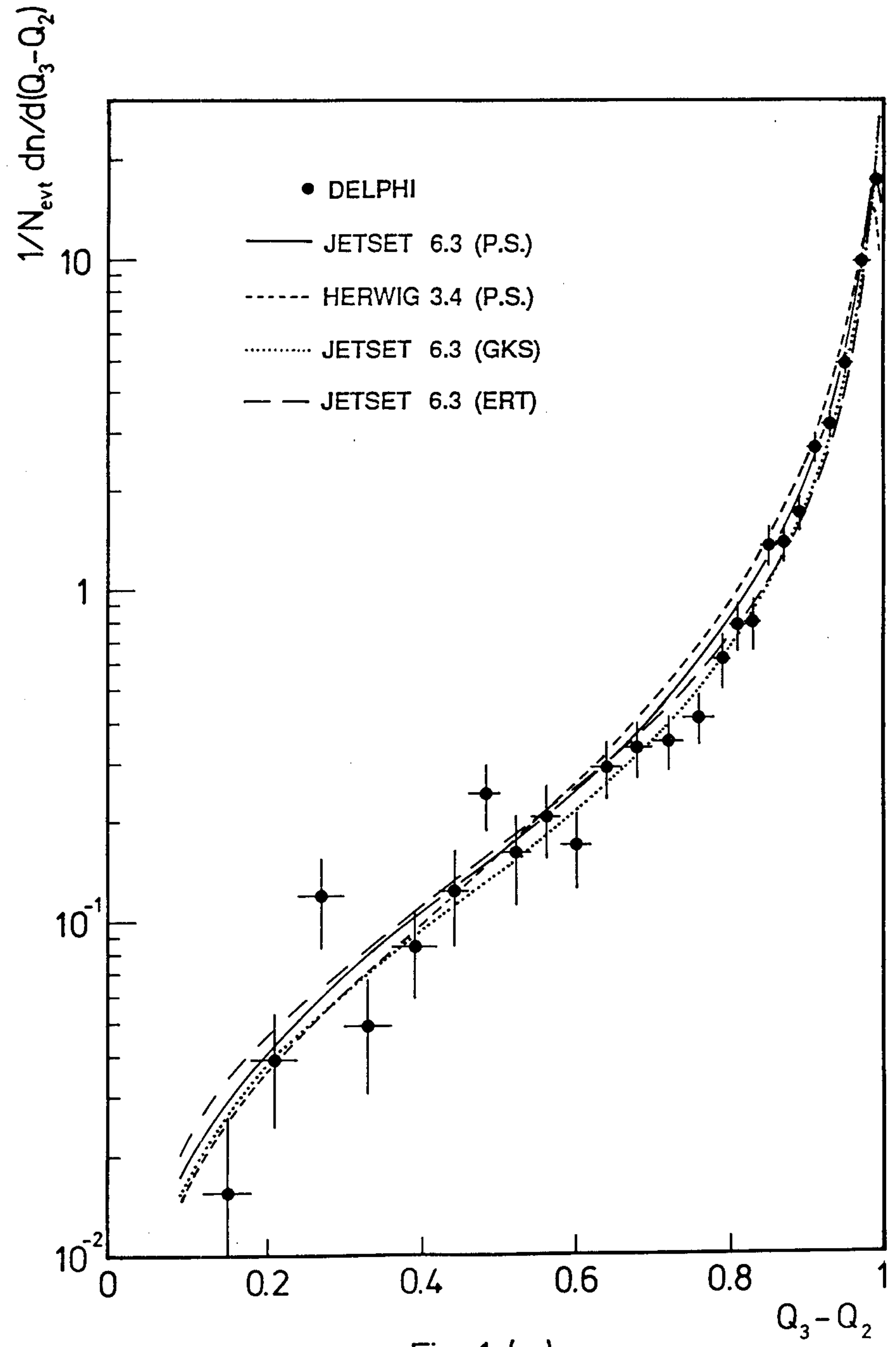

Fig. $1(c)$ 


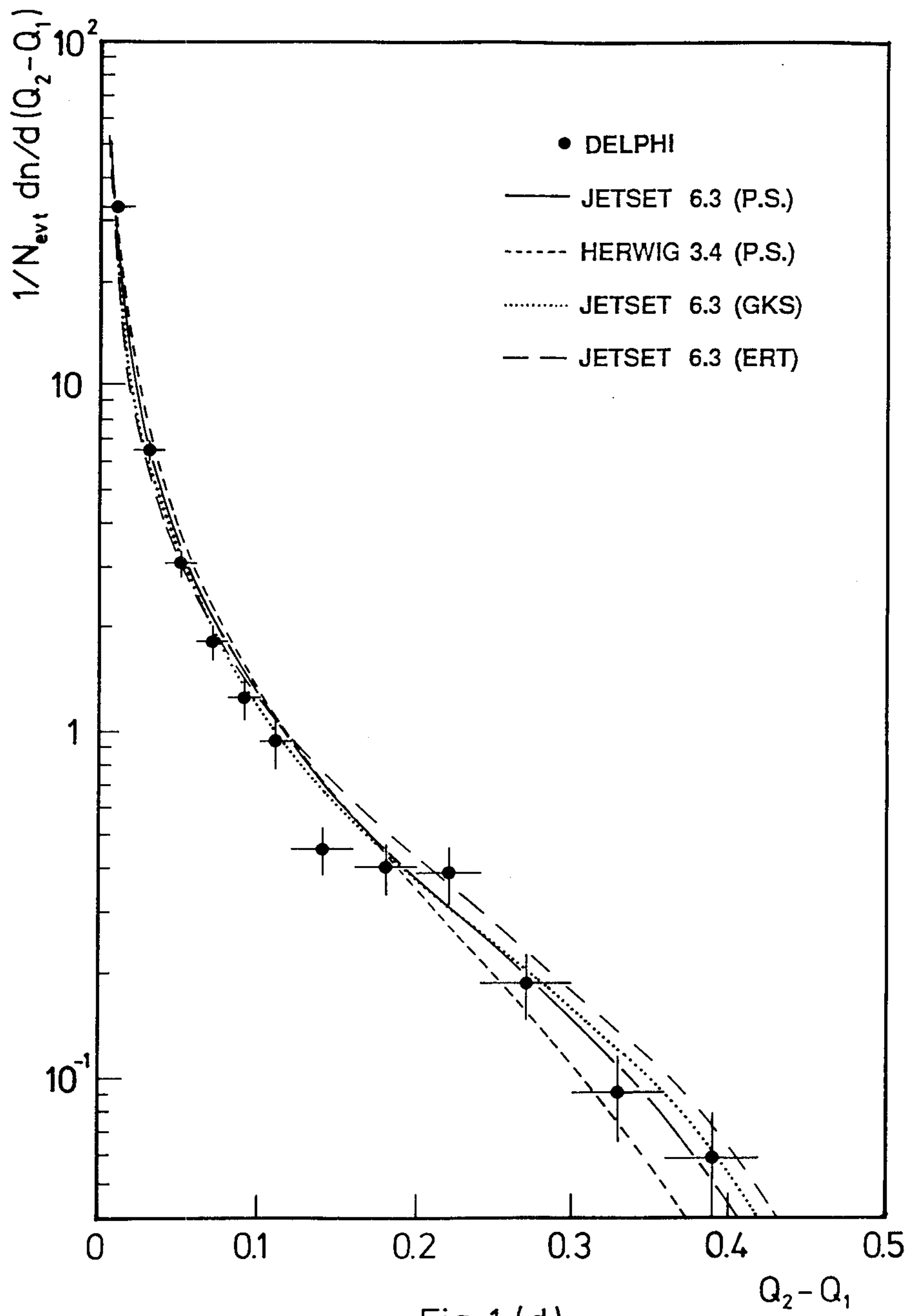

Fig. 1 (d) 


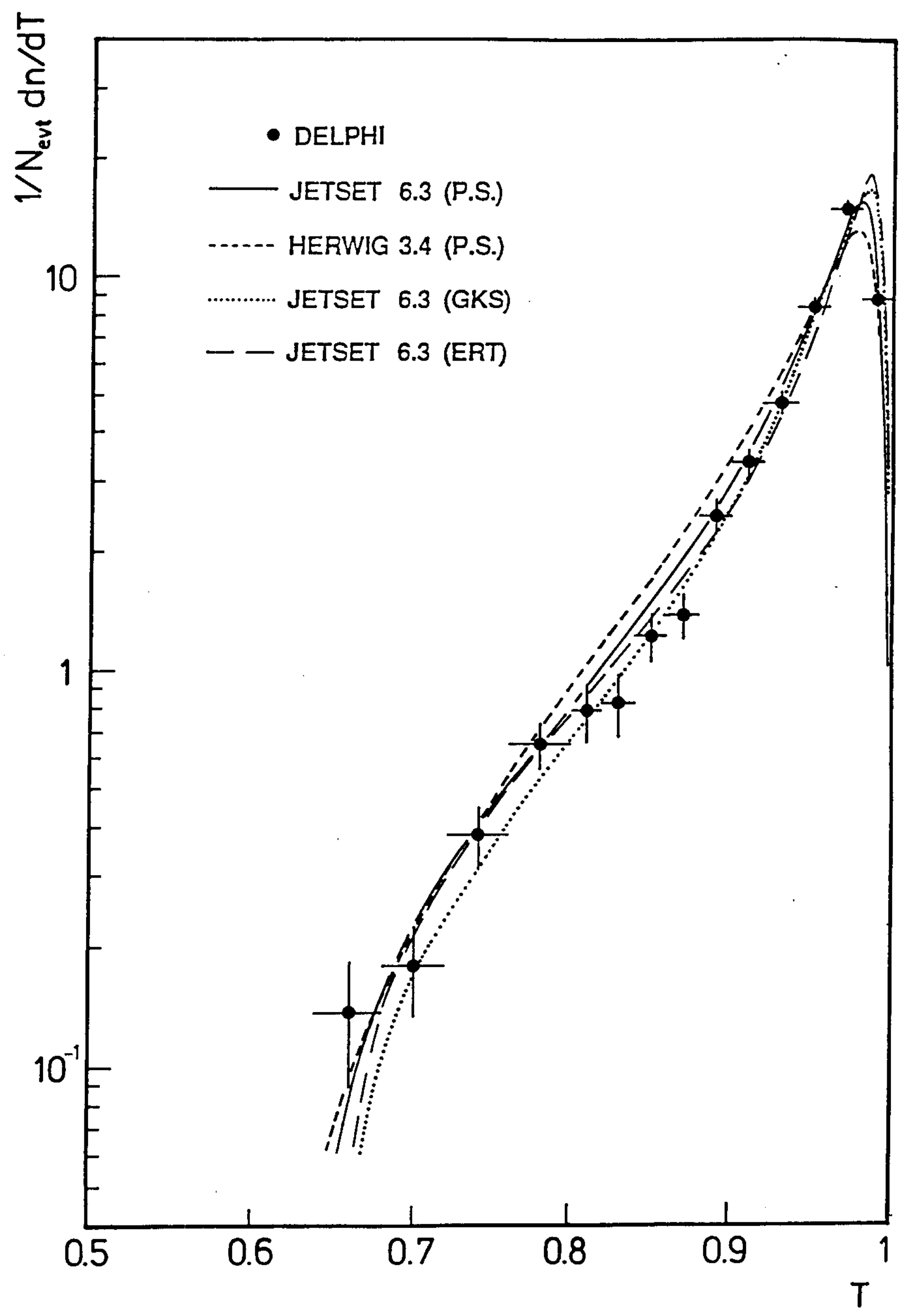

Fig. 2 (a) 


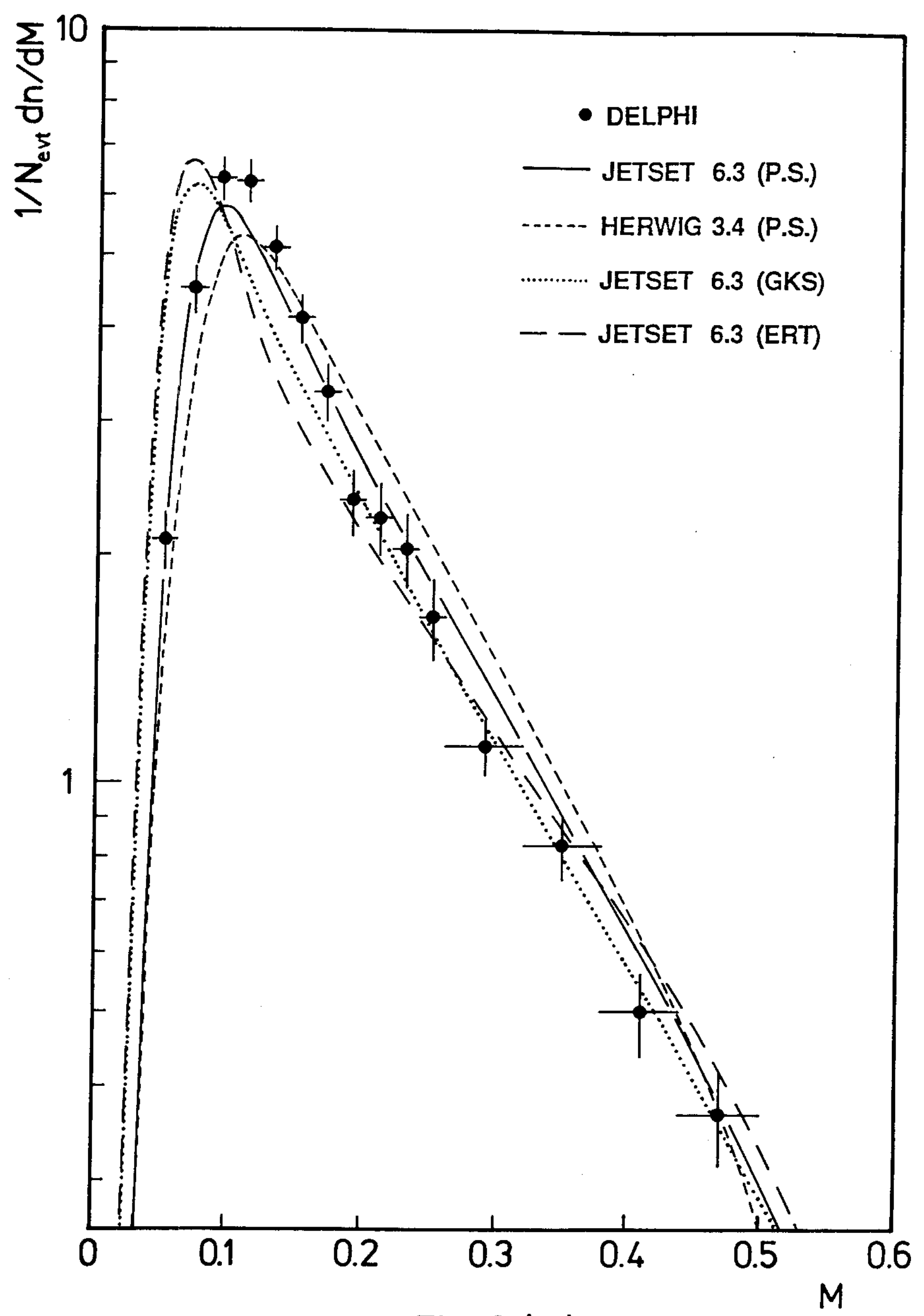

Fig. 2 (b) 


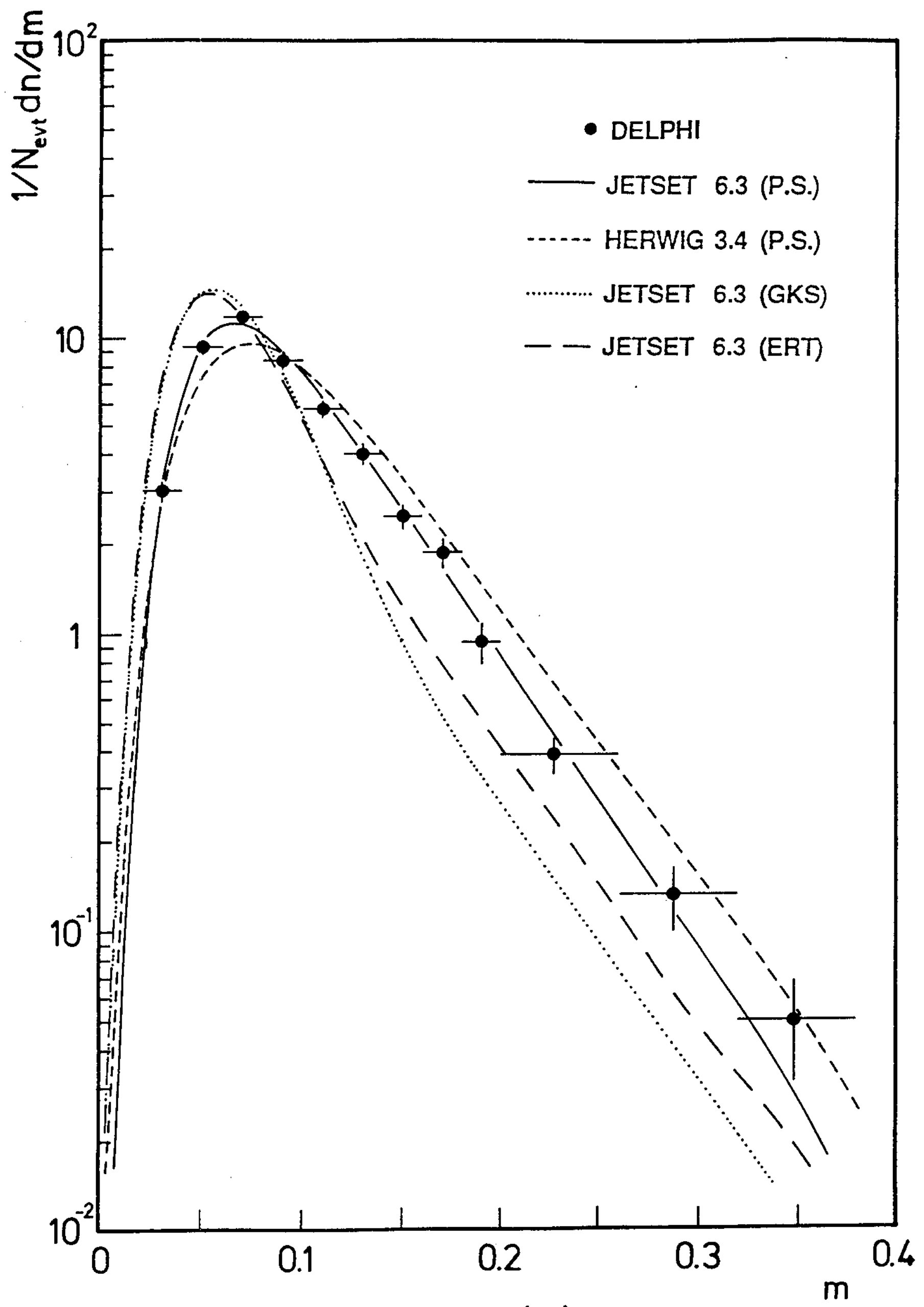

Fig. 2 (c) 


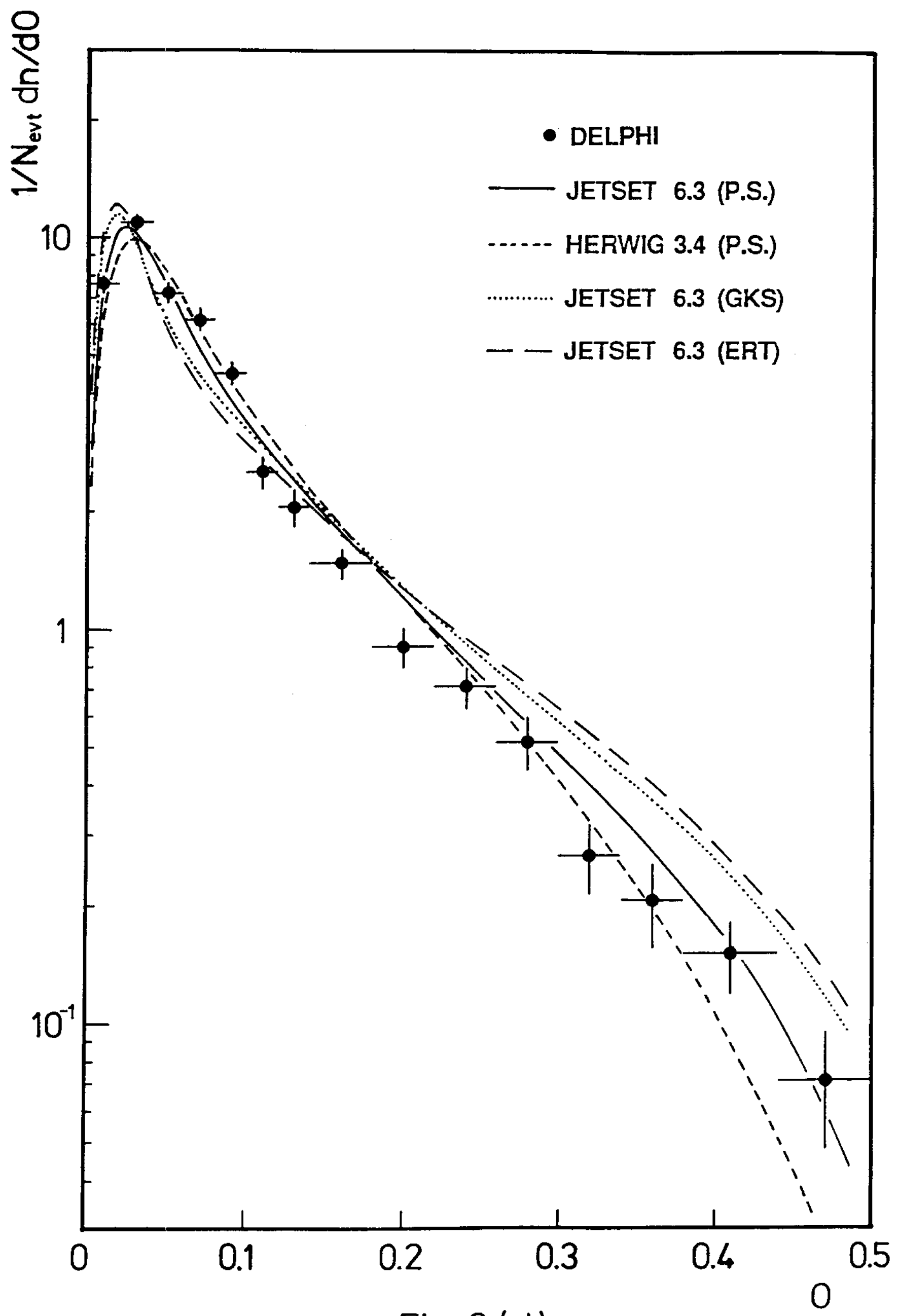

Fig. 2 (d) 


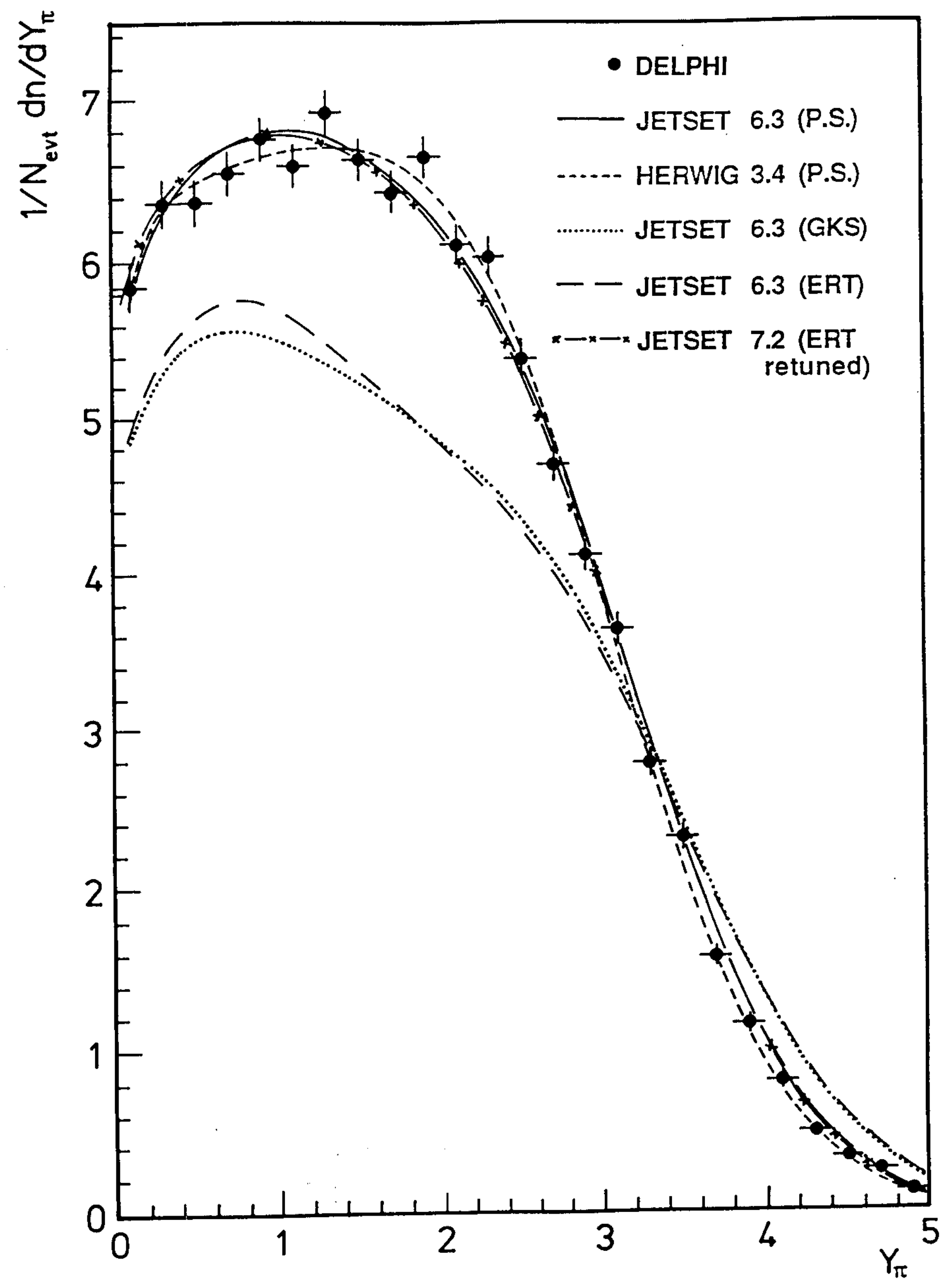

Fig. 3 (a) 


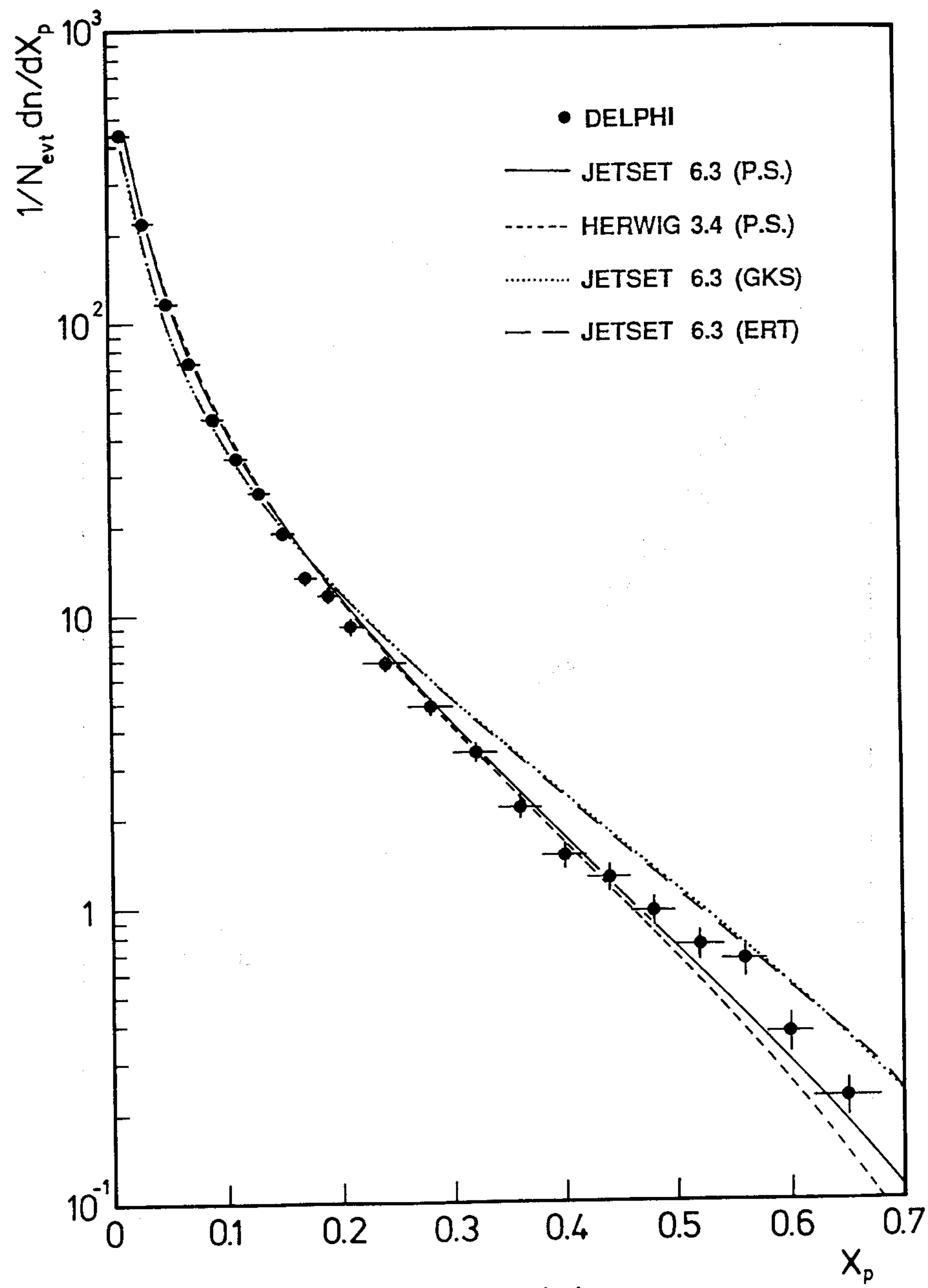

Fig. 3 (b) 


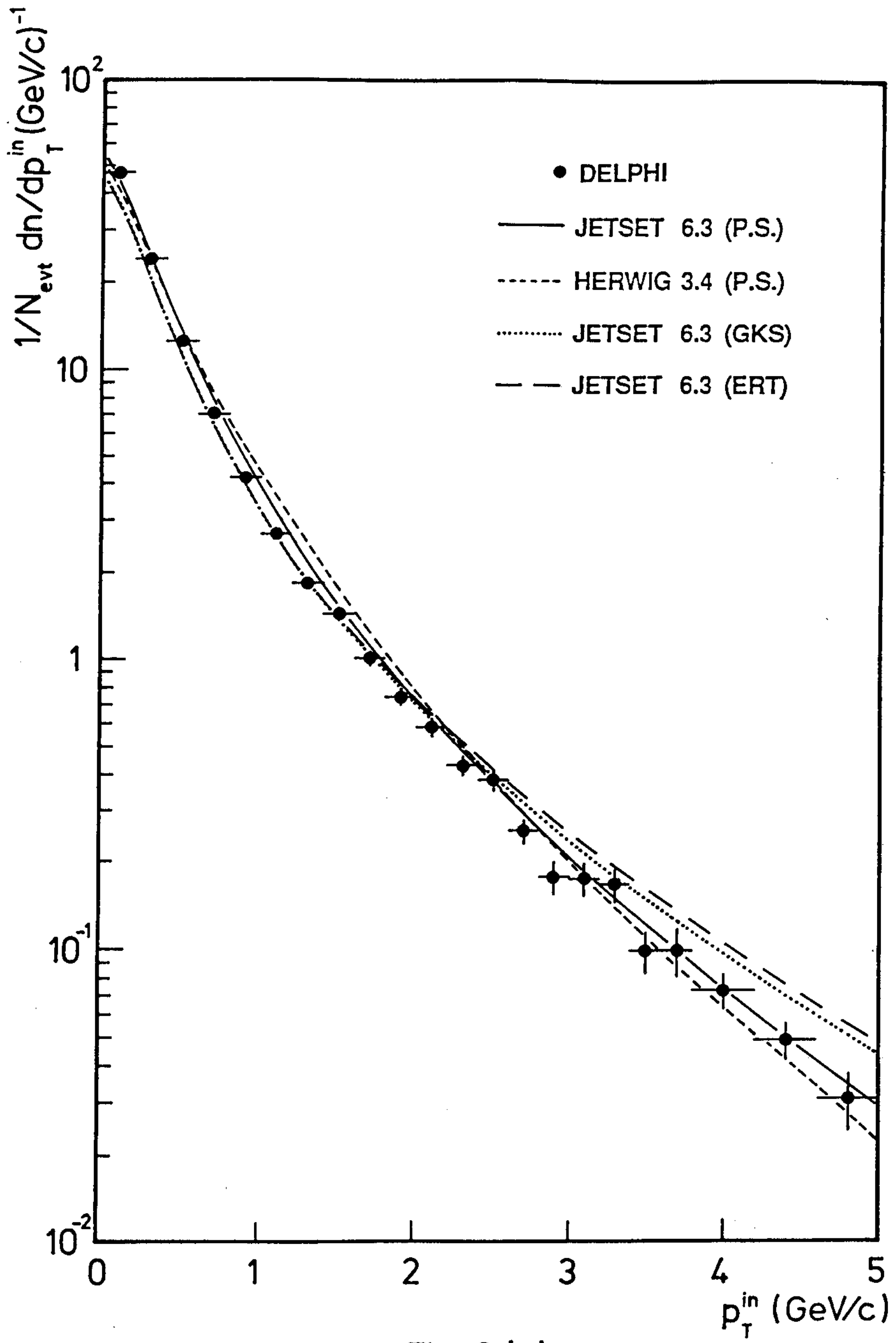

Fig. $3(c)$ 


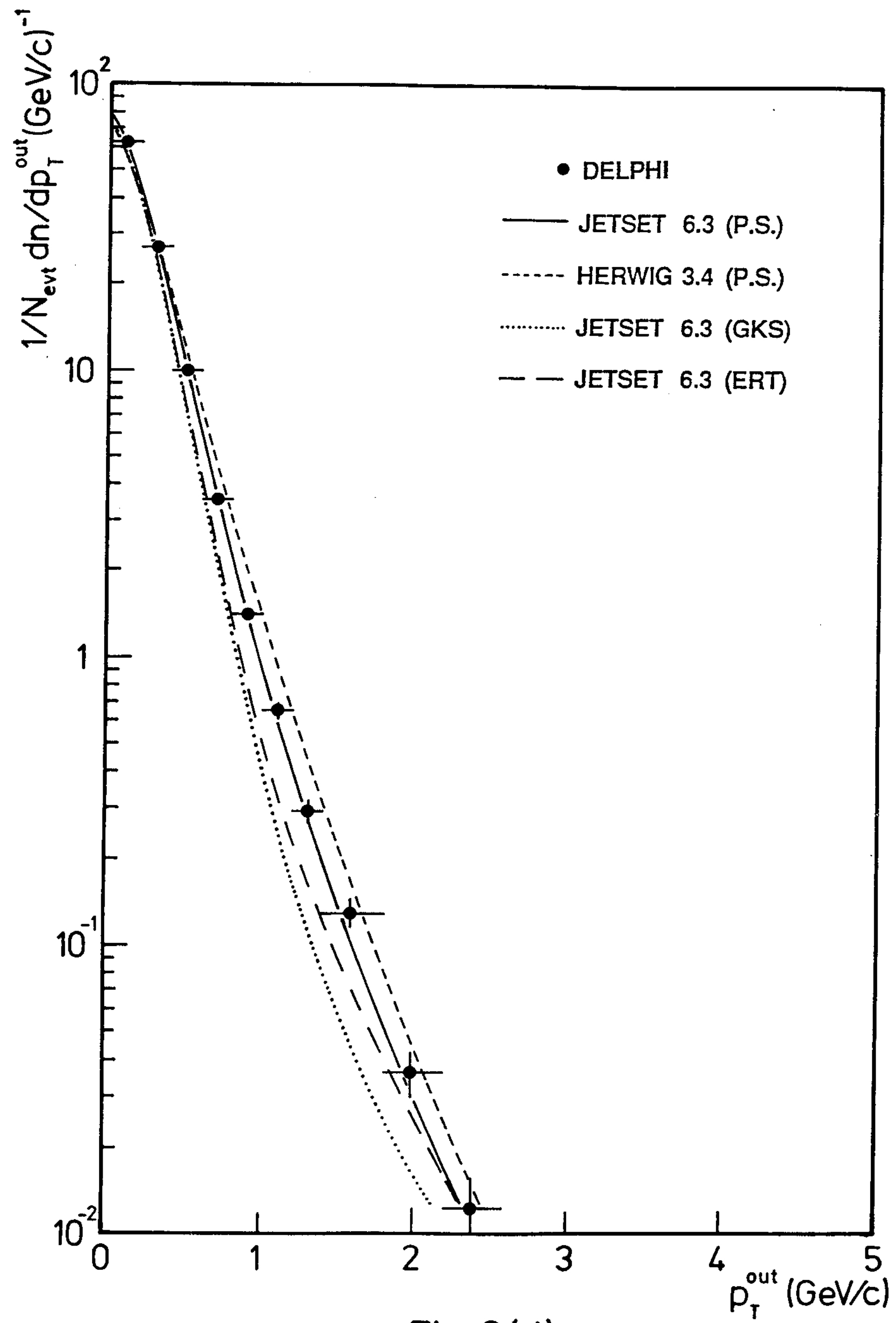

Fig. $3(d)$ 

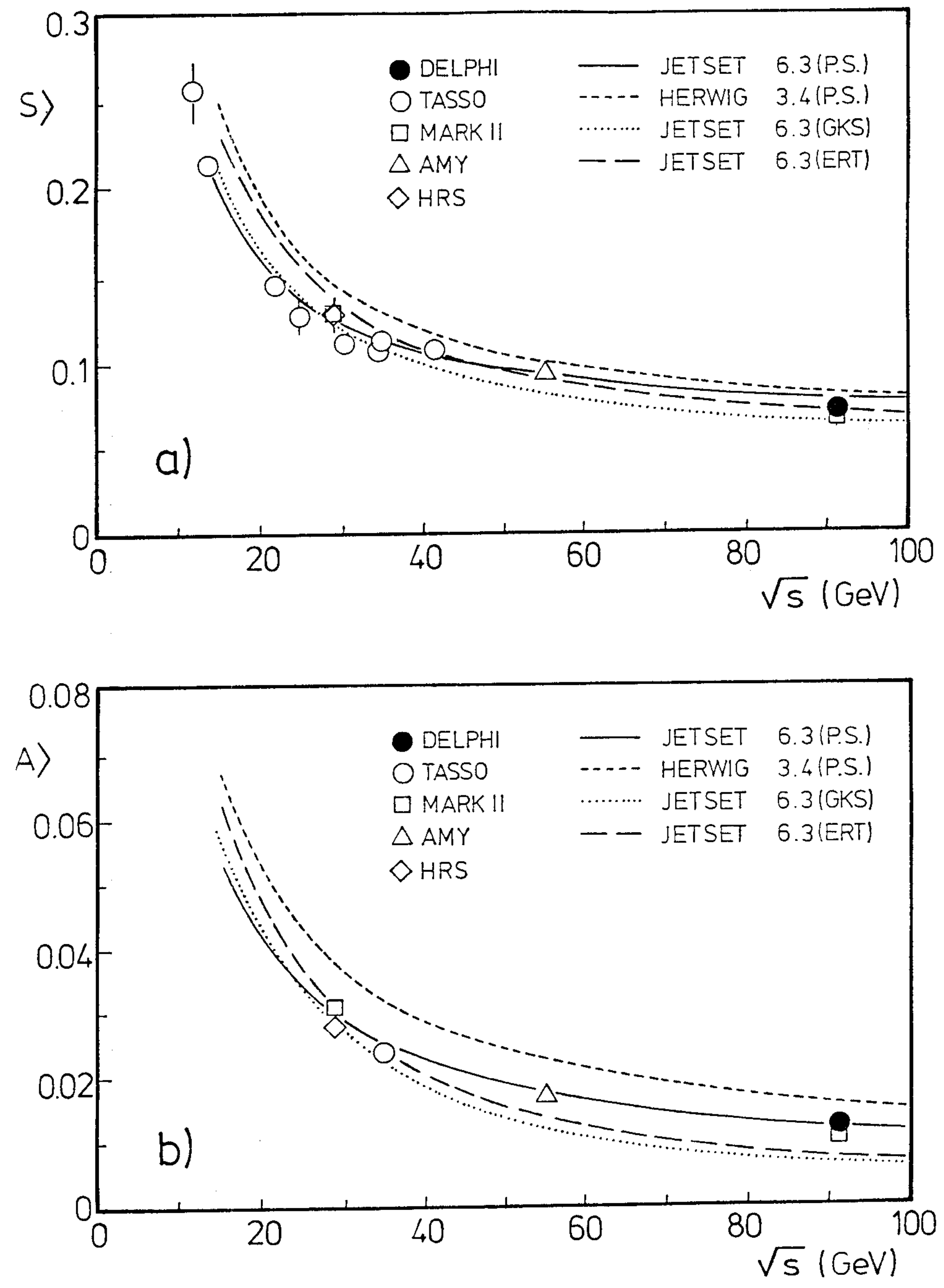

Fig. 4 

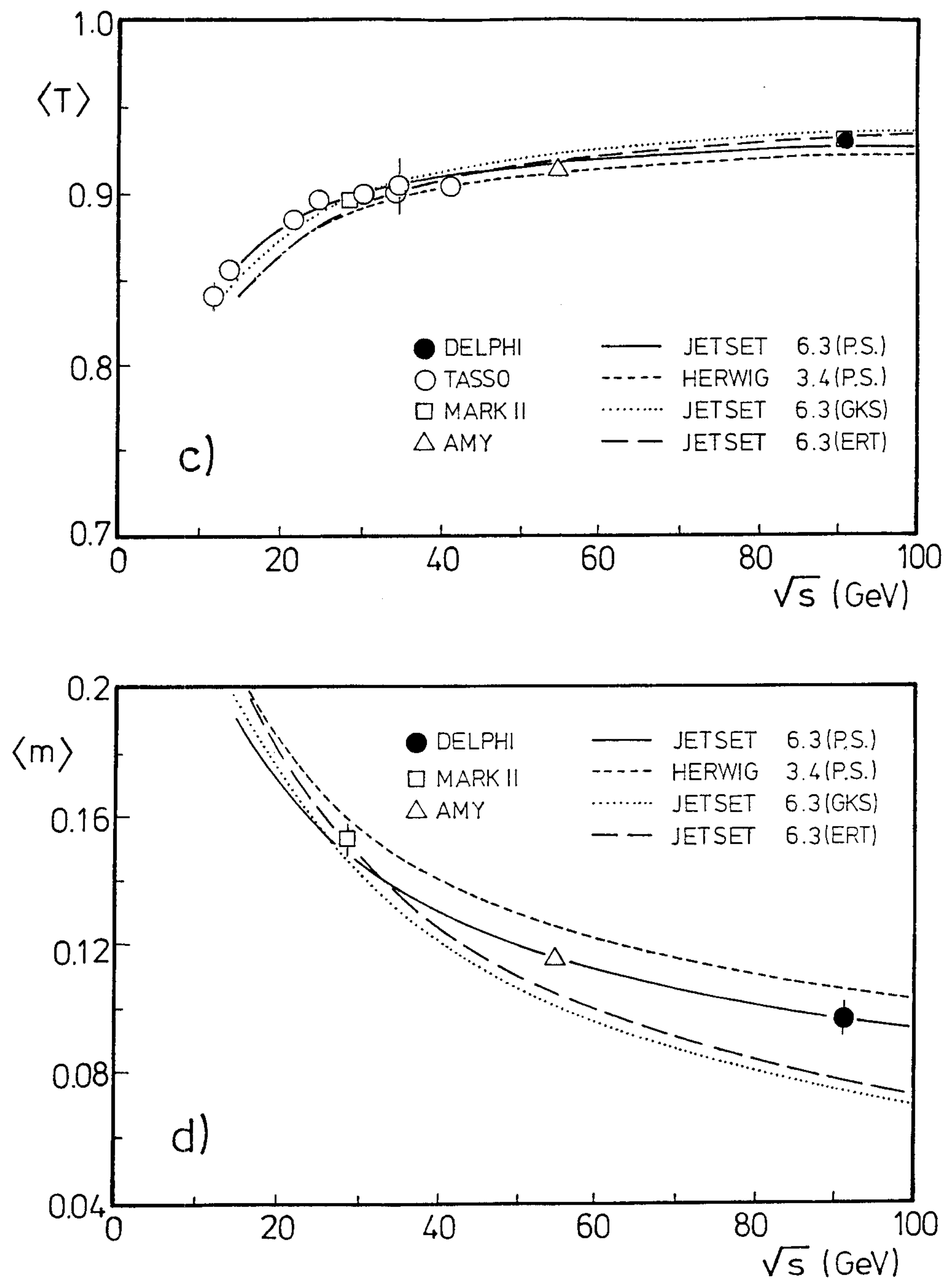

Fig. 4 

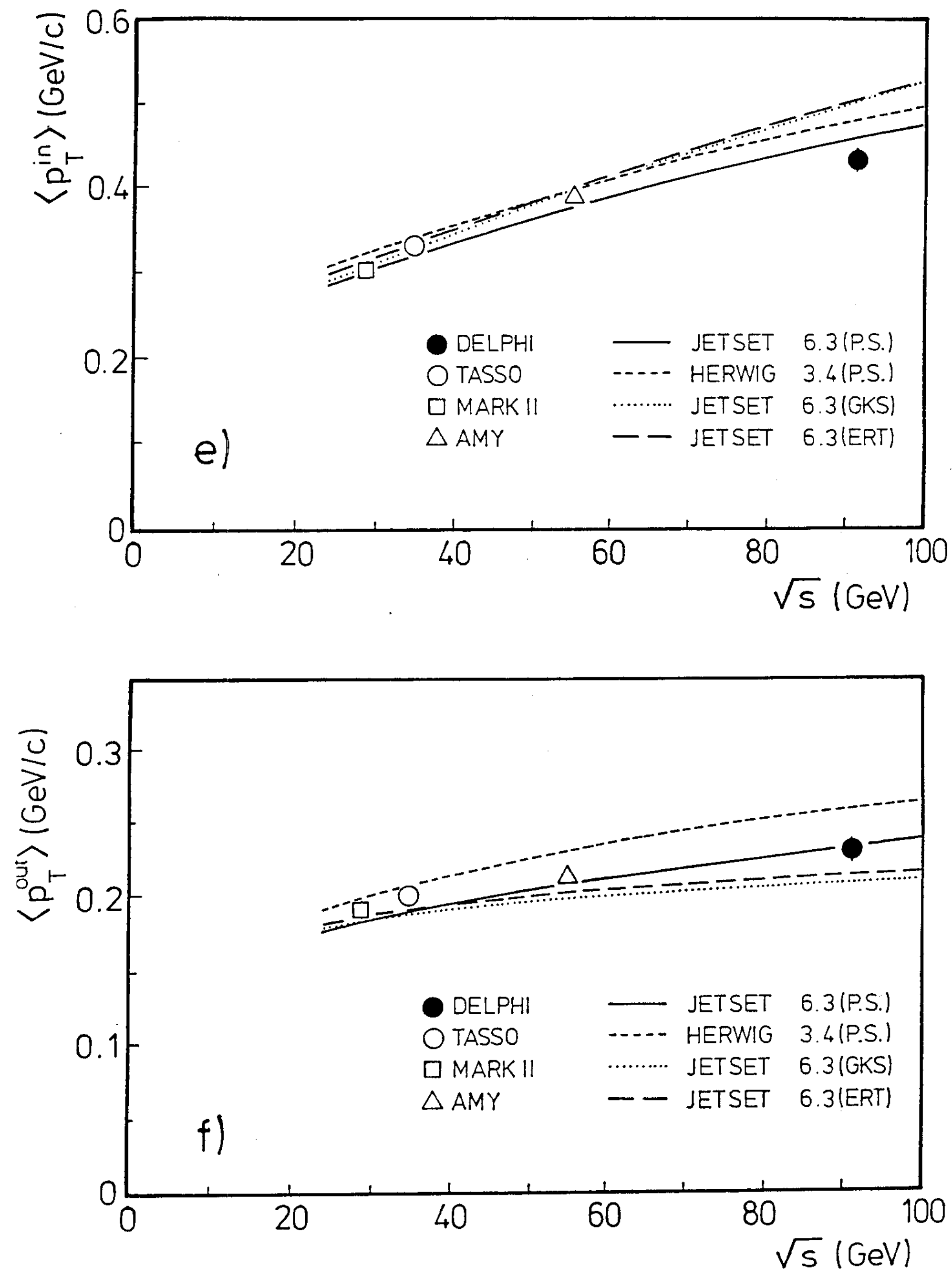

Fig. 4 